\title{
Characterization of Supersonic Gas Jets for High-Resolution Laser Ionization Spectroscopy of Heavy Elements
}

\author{
A. Zadvornaya, ${ }^{*}$ P. Creemers, K. Dockx, R. Ferrer, L. P. Gaffney, ${ }^{\dagger}$ W. Gins, C. Granados, ${ }^{\dagger}$ \\ M. Huyse, Yu. Kudryavtsev, M. Laatiaoui, E. Mogilevskiy, ${ }^{\ddagger}$ S. Raeder, ${ }^{\S}$ S. Sels, P. Van den Bergh, \\ P. Van Duppen, M. Verlinde, and E. Verstraelen \\ KU Leuven, Instituut voor Kern- en Stralingsfysica, Celestijnenlaan 200D, B-3001 Leuven, Belgium \\ M. Nabuurs and D. Reynaerts \\ KU Leuven, Department of Mechanical Engineering, \\ Celestijnenlaan 200F, B-3001 Leuven, Member of Flanders Make, Belgium \\ P. Papadakis $\|$ \\ University of Jyvaskyla, Department of Physics, P.O. Box 35, FI-40014 University of Jyvaskyla, Finland
}

(Received 12 April 2018; revised manuscript received 9 August 2018; published 12 October 2018)

\begin{abstract}
The method of laser spectroscopy in supersonic gas jets was proposed for high-resolution and highefficiency in-gas laser ionization and spectroscopy studies of short-lived nuclei. The flow properties of such supersonic gas jets have been characterized under off-line conditions. Planar laser-induced fluorescence spectroscopy of seeded copper atoms has been applied to nonintrusively measure velocity, temperature, and relative density profiles of gas jets formed by different de Laval nozzles. For validation, planar laserinduced fluorescence spectroscopy was applied on supersonic free jets with well-known flow parameters. The performance of the in-gas-jet laser spectroscopy method is determined by the achievable spectral resolution, which requires an optimization and a precise manufacturing of the nozzle inner contour as well as a pressure matching of the background medium at the nozzle exit. Our studies now enable a thorough understanding and quantification of these requirements and a determination of the final performance of the in-gas-jet method. Additionally, a comparison between the experimental results and the numerical calculations was performed for the temperature, velocity, and Mach number profiles of underexpanded and quasiuniform jets formed by a de Laval nozzle.
\end{abstract}

DOI: 10.1103/PhysRevX.8.041008

Subject Areas: Fluid Dynamics, Nuclear Physics

\section{INTRODUCTION}

Experimental laser spectroscopy studies of the actinides give access to nuclear ground- and isomeric-state properties, such as mean-square charge radii, magnetic dipole and

\footnotetext{
*Sasha.Zadvornaya@kuleuven.be alexandra.zadvornaya@gmail.com

†Present address: CERN, CH-1211 Genève, Switzerland.

*Present address: Faculty of Mechanics and Mathematics, Lomonosov Moscow State University, Leninskie gory, 1, 119992 Moscow, Russia.

${ }^{\S}$ Present address: GSI Helmholtzzentrum für Schwerionenforschung GmbH, Planckstrasse 1, D-64291 Darmstadt, Germany.

${ }^{\|}$Present address: Oliver Lodge Laboratory, University of Liverpool, Liverpool, L69 7ZE, United Kingdom.

Published by the American Physical Society under the terms of the Creative Commons Attribution 4.0 International license. Further distribution of this work must maintain attribution to the author(s) and the published article's title, journal citation, and DOI.
}

electric quadrupole moments, and spins, in a nuclear model independent way, provided the atomic physics is known [1]. The information on these observables is essential to validate nuclear models applied in the heavy element region [2,3] and to increase their predictive power, for instance, to locate the anticipated island of enhanced stability [4]. In this way, laser spectroscopy is complementary to nuclear spectroscopy, which until now is the only technique studying the heaviest elements $[5,6]$.

Up until now the heaviest synthesized element is ${ }^{294} \mathrm{Og}$ $(Z=118)$ with a determined value of its production cross section at the subpicobarn level $[7,8]$. On the other hand, the heaviest element with a measured ionization potential is ${ }^{256} \operatorname{Lr}(Z=103)$ [9], whereas that with an observed groundstate atomic transition is ${ }^{254}$ No $(Z=102)$ [10]. The knowledge of basic nuclear ground-state properties is very limited even in the actinide region $(89 \leq Z \leq 103)$ due to a restricted spectral resolution in the currently applied laser spectroscopy techniques and to a number of experimental challenges. Artificially synthesized heavy nuclei usually 
have short lifetimes, e.g., $T_{1 / 2}\left({ }^{215} \mathrm{Ac}\right)=170 \mathrm{~ms}$. Moreover, extended isotopic chains of heavy elements $(Z \geq 89)$ are difficult to synthesize: nuclide production via fusion evaporation reactions is feasible only for certain projectile-target combinations $[11,12]$ with isotope yields dropping fast when moving farther from the "valley of stability." In general, as no tabulated spectral lines exist for the transfermium elements, extensive broadband scans within spectral ranges proposed by atomic theory are required to identify atomic transitions. These facts turn efficient separation and study of the isotopes of interest into a challenging task. A comprehensive study of the groundand excited-state properties of the actinides thus calls for a high-resolution and high-sensitive experimental technique.

A number of laser spectroscopy techniques for unstable nuclei are used, including collinear and in-source approaches [1]. Collinear fluorescence and resonance ionization spectroscopy provide quasi-Doppler-free resolution, but require radioactive ion beam production rates of at least 100 and 20 particles/s, respectively (see, e.g., Refs. $[13,14])$. The latter rates, however, have so far not been reached in the heavy element region. In-source laser resonance ionization spectroscopy in a hot cavity has a sensitivity down to 0.1 atoms/s, but the accessible spectral resolution is limited to a few $\mathrm{GHz}$ by the temperature associated broadening due to target and an ion source's temperature of above $2000 \mathrm{~K}$ [15]. Moreover, this technique cannot be used with the refractory elements and in its current implementation is limited to elements with $Z<92$.

The in-gas laser ionization and spectroscopy (IGLIS) technique has been in continuous development at KU Leuven during the past three decades [16], with extensive work published on the characterization and optimization of its performance [17-19]. In-gas-cell laser spectroscopy experiments were performed on neutron deficient ${ }^{57,59} \mathrm{Cu}$ [20], ${ }^{97-101} \mathrm{Ag}$ [21], and ${ }^{212-215} \mathrm{Ac}$ [22] at the Leuven Isotope Separator On-Line (LISOL) facility coupled to the CYCLONE accelerator of the Centre de Ressources du Cyclotron at Louvain-la-Neuve (Belgium). Information about ground-state properties of these nuclei, such as nuclear magnetic dipole moments, isotope shifts, and mean-square charge radii, were obtained. Yet, limited spectral resolution due to collision and temperature related broadening mechanisms made it impossible to extract other basic ground- and isomeric-state properties, such as nuclear electric quadrupole moments and nuclear spins.

With the in-gas-jet method, however, the spectral resolution and the selectivity can be significantly improved, while maintaining a high efficiency. In this method, ionization of the isotopes of interest takes place in supersonic gas jets with flow velocities exceeding the local speed of sound, i.e., with a Mach number $M>1$. Uniform and long gas jets, formed under certain experimental conditions by an axisymmetric convergent-divergent de Laval nozzle, are characterized by a low-density and low-temperature gas medium with a well-defined Mach number along the central jet line. Low densities and temperatures result in a significant reduction of the associated collision and temperature broadening mechanisms in comparison to those measured in the gas cell. The implementation of in-gas-jet resonance laser ionization increases the spectral resolution by more than 1 order of magnitude in comparison to in-gas-cell ionization spectroscopy [23], as recently demonstrated for the actinium isotopes in the proximity and at the $N=126$ neutron shell closure [24]. Despite the not fully optimized experimental conditions, the enhancement in spectral resolution obtained in the latter experiments allowed the extraction of the nuclear electric quadrupole moment and nuclear spin for the ${ }^{214,215} \mathrm{Ac}$ isotopes. As an example, the inset in Fig. 1 shows the measured spectrum of the ${ }^{214} \mathrm{Ac}$ isotope with a spectral linewidth of about $400 \mathrm{MHz}$ along with the simulated spectrum for the projected working conditions at $M=10$ resulting in a spectral linewidth of $\sim 100 \mathrm{MHz}$.

In order to further investigate the limits of the attainable spectral resolution of the in-gas-jet method, an in-depth understanding and characterization of the gas jet formation is required, especially as the in-gas-jet method will be implemented in the next generation of radioactive ion beam (RIB) facilities, such as $\mathrm{S}^{3}$-LEB at SPIRAL2 of GANIL (France) [25], MARA separator at University of Jyväskylä (Finland) [26], GALS setup at JINR (Russia) [27], KISS and PALIS setups at RIKEN RIBF (Japan) [28,29], and SHIP setup at GSI (Germany), for the laser spectroscopy studies of radioactive nuclei. An overview of the isotopes that can be studied with the in-gas-jet method, considering their production cross sections and assuming a primary intensity of a heavy ion beam of $10 \mathrm{p} \mu \mathrm{A}$, an efficiency of the method of $10 \%$, and yields of 0.1 p.p.s. extracted from the gas cell, is shown in Fig. 1.

A number of flow characterization techniques have been developed to gain insight into the processes taking place in fluid (liquid and gaseous) flows, with the first works performed by Reynolds and Prandtl and the breakthrough experiments of Mach [30]. An in-depth characterization of the flow parameters is feasible with modern flow field diagnostic techniques. The Rayleigh scattering technique provides simultaneous measurements of the temperature, velocity, and density in unseeded flows [31] and, moreover, it allows time-resolved diagnostic [32]. However, this technique requires high densities of the scattering species and gives only a point measurement of the flow field parameters, thus requiring the probe volume to be moved from point to point to cover the full flow field. Besides that, a meticulous elimination of the light scattered from particles in the gas or nearby surfaces is required, since this light has the same wavelength as the Rayleigh signal. Electron beam-excited fluorescence enables the visualization and the extraction of temperature, velocity, and density flow fields, as demonstrated, e.g., for supersonic free jets 


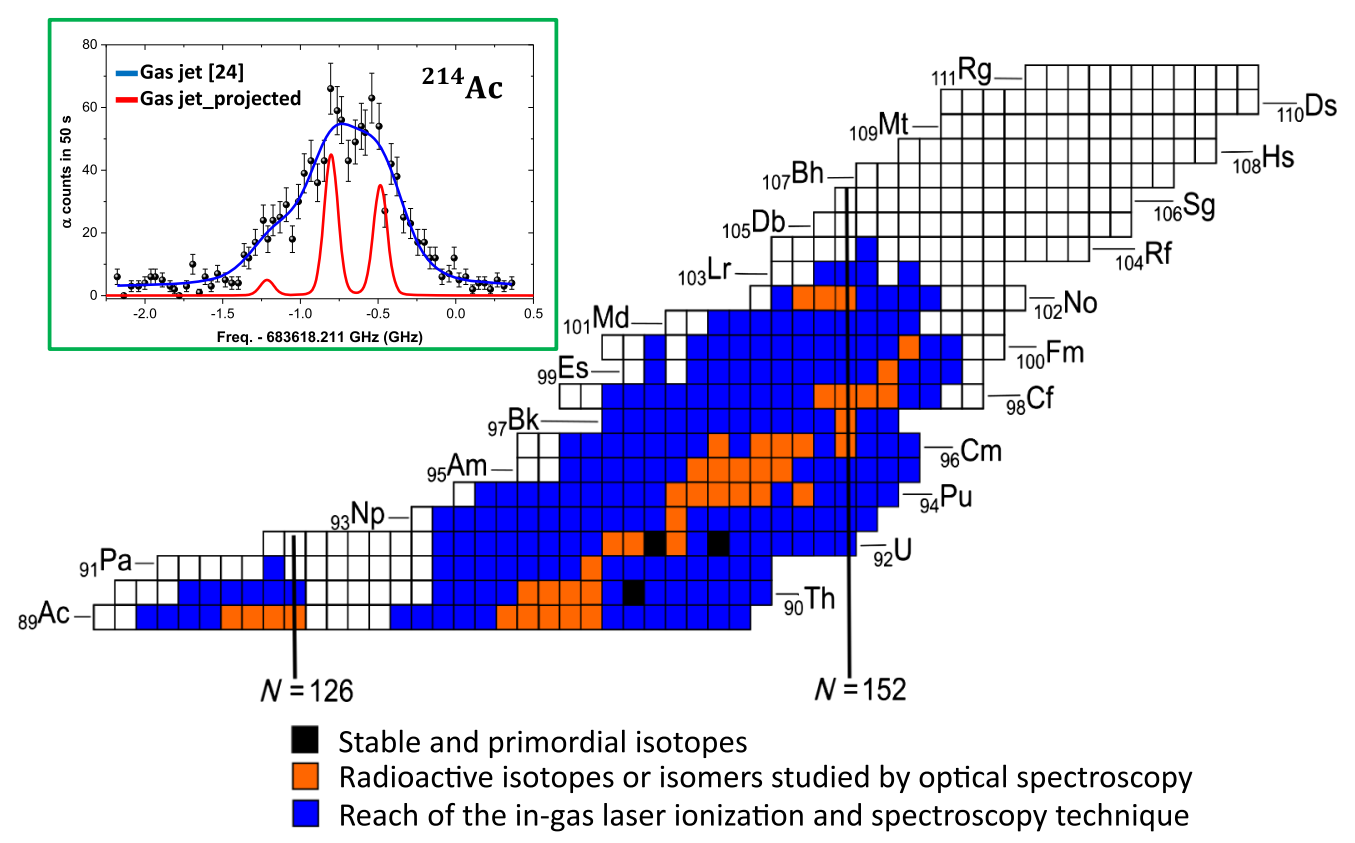

FIG. 1. Enlargement of the chart of nuclides in the actinides region: stable and primordial isotopes are shown by black squares, radioactive isotopes already studied with laser spectroscopy are shown in brown [1]. The elements which can be produced in sufficient amounts for the IGLIS technique are highlighted in blue with the prediction based on experimental and calculated cross sections and assumptions of a primary beam intensity of $10 \mathrm{p} \mu \mathrm{A}$, an efficiency of the method of $10 \%$ and yields of 0.1 p.p.s. extracted from the gas cell. This corresponds to a lower limit in production cross section of $50 \mathrm{nb}$. In the inset, the hyperfine structure of the $6 d 7 s^{2}{ }^{2} D_{3 / 2} \rightarrow$ $6 d 7 s 7 p^{4} P_{5 / 2}^{0}$ transition in atomic ${ }^{214} \mathrm{Ac}$ as measured using the in-gas-jet method is shown (black dots) with the blue curve representing the best fit using a Voigt line profile. The frequency detuning is shown with respect to the center of gravity of the ${ }^{227} \mathrm{Ac}$ isotope. The expected performance of the in-gas-jet method at Mach number $M=10$ is shown with the red curve.

and jets formed by de Laval nozzles using helium, neon, argon, and xenon $[33,34]$. Although electron beam-excited fluorescence is suited for the characterization of lowdensity flows [35], it cannot be applied for the extraction of the spectral broadening of the seeded atoms, since it characterizes the properties of the carrier gas. Another method, that has been applied for the visualization of helium and argon jets, is the fluorescence excitation by an electrical discharge $[36,37]$. On the other hand, planar laser-induced fluorescence (PLIF) spectroscopy [38] makes it possible to extract two-dimensional temperature flow fields of jets (demonstrated for helium, argon, and heated air) and flames (methane-air) seeded with molecular species $\left(\mathrm{NO}, \mathrm{O}_{2}\right)$ [39-41]. Extraction of two-dimensional velocity flow fields has been demonstrated for argon, helium, and nitrogen jets seeded with molecular species $\left(\mathrm{NO}, \mathrm{I}_{2}\right)$ $[39,42,43]$. Moreover, the PLIF technique was applied for density mapping of plasma jets seeded with copper atoms and of laser-ablated copper plasma plumes [44,45].

In this paper, we present the first application of atomic PLIF spectroscopy for the characterization of the local flow parameters (velocity, temperature, and relative density) of supersonic jets formed by de Laval nozzles. These studies were performed at the recently commissioned IGLIS laboratory [46], where PLIF spectroscopy, utilizing the fluorescence light emitted by the stable ${ }^{63,65} \mathrm{Cu}$ isotopes seeded into an argon flow, was applied. After a description of the IGLIS laboratory with a dedicated PLIF spectroscopy setup, the PLIF spectroscopy studies are validated using supersonic free jets. The results of characterization studies of the supersonic jets are discussed and, finally, the future prospects of the in-gas-jet method at the next generation of RIB facilities are presented.

\section{EXPERIMENTAL METHOD}

\section{A. IGLIS laboratory \\ 1. General description}

A full description of the IGLIS laboratory is given in Ref. [46]. Here, only the details related to the PLIF experiments are described thoroughly with a schematic layout of a PLIF spectroscopy setup shown in Fig. 2. Within IGLIS studies, the resonance two-step laser ionization via an intermediate excited state takes place at the intersection of the first and second step laser beams with the jet, making it possible in this way to choose the ionization volume and to avoid ionization of the atoms inside the gas cell. One of the laser beams is directed longitudinally with respect to the gas jet, while the other one is in the transverse direction. To provide high spectral resolution and efficiency of the in-gas-jet method, the formation of a long collimated jet of a high Mach number is required. To guarantee a 


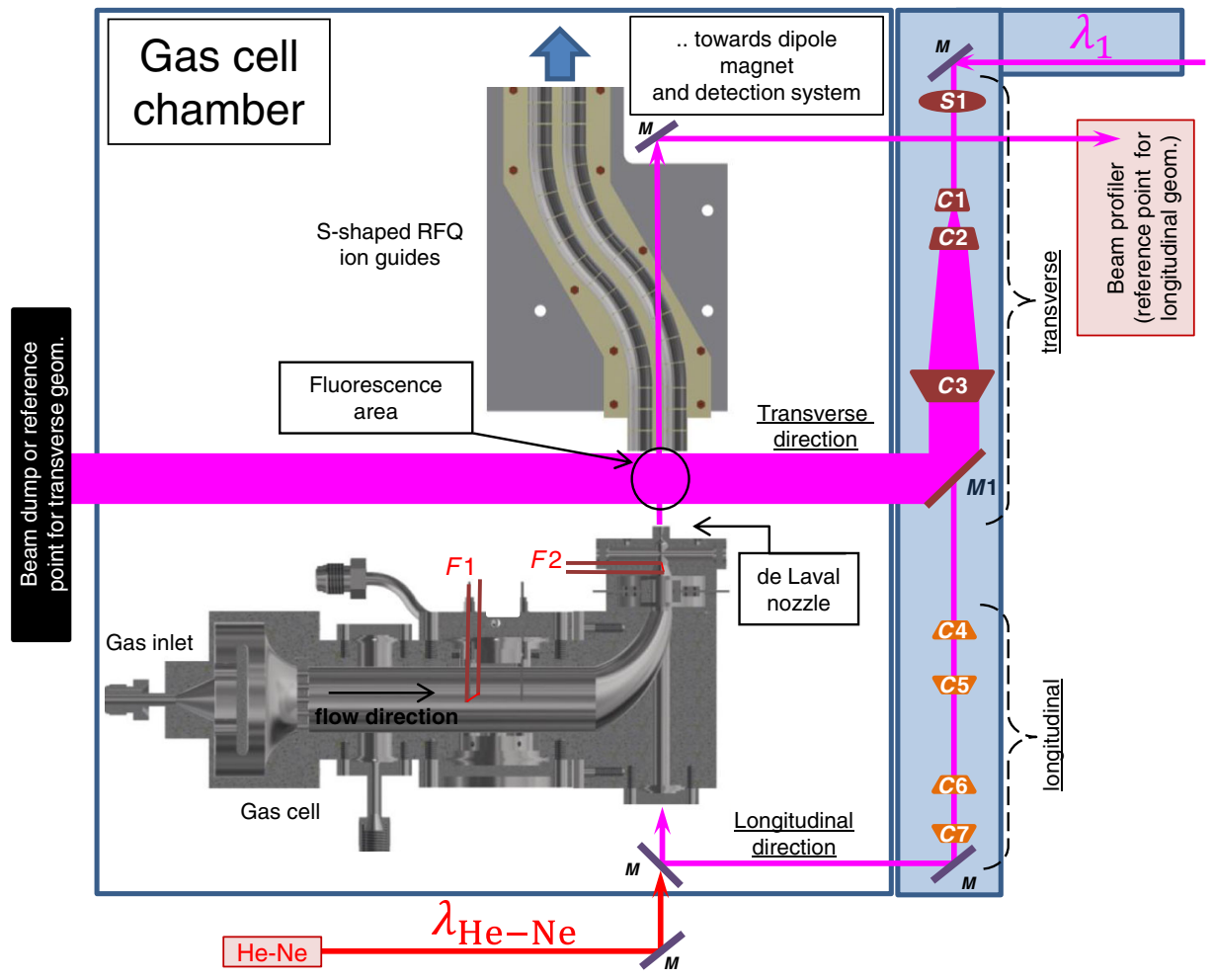

FIG. 2. Layout of the PLIF spectroscopy setup (not to scale). A more detailed layout of the IGLIS laboratory is given in Ref. [46]. Here, $\lambda_{1}$ and $\lambda_{\mathrm{He}-\mathrm{Ne}}$ denote a laser used for PLIF experiments $\left(\lambda_{1}=327.40 \mathrm{~nm}\right)$ and a helium-neon laser, respectively. $F 1$ and $F 2$ indicate two tested filament positions. An ICCD camera used in PLIF experiments was oriented normally to the view shown here. $M$ denotes UV mirrors. Transverse direction: $S 1$ is a spherical lens, $C 1$ and $C 2$ are plano-concave cylindrical lenses, and $C 3$ is a plano-convex cylindrical lens. Longitudinal direction: $(C 4+C 5)$ and $(C 6+C 7)$ are two sets of (plano-concave + plano-convex) cylindrical lenses.

complete temporal overlap between the pulsed laser radiation and the atoms in the continuous high-velocity flow, and thus ensure high ionization efficiency of the in-gas-jet ionization method, the width of the transverse laser beam should be $55 \mathrm{~mm}$ for an $\operatorname{argon}$ jet $(v=550 \mathrm{~m} / \mathrm{s}$ at a temperature inside the gas cell $T_{0}=300 \mathrm{~K}$ ) and for a laser repetition rate of $10 \mathrm{kHz}$. The laser-produced ions are confined and transported through a set of radio frequency quadrupole (RFQ) ion guides, accelerated to an energy of $40 \mathrm{keV}$, and sent towards a dipole magnet for isotope separation according to their mass-to-charge ratio. Finally, isotopically pure ion beams are measured at a detection station comprising a Faraday cup, properly biased to ensure a correct current read-out, and a multichannel plate (MCP) detector.

\section{Gas cell chamber}

The PLIF experiments were performed with the stable copper ${ }^{63,65} \mathrm{Cu}$ isotopes. The isotopes were produced inside a stainless steel gas cell by resistively heating a copper filament and were fed into a high-purity argon flow inside the gas cell with stagnation pressure $P_{0}$ in a range from 250 to 350 mbar. A gas cell design was optimized to reduce transportation losses and to ensure a fast evacuation from the gas cell of species with potentially short lifetimes [47]. Two filaments ( $F 1$ and $F 2$ in Fig. 2) installed at different positions inside the gas cell were tested, resulting in different stagnation temperatures $T_{0}$ close to the nozzle position. Higher temperature $T_{0}$ was obtained for the case of filament $F 2$ (see Secs. III and IV).

The argon gas from Air Liquide $\left[\mathrm{H}_{2} \mathrm{O}<0.5 \mathrm{ppm} ; \mathrm{H}_{2}\right.$, $\mathrm{O}_{2}, \mathrm{CNH}(\mathrm{M}), \mathrm{CO}$ and $\left.\mathrm{CO}_{2}<0.1 \mathrm{ppm}\right]$ was purified in a gas purifier PS4-MT3-R-2 (MonoTorr) down to the ppb level and then sent via a clean, well-conditioned gas line towards the gas cell. The copper atoms were transported by the gas flow towards a de Laval nozzle installed at the gas cell exit and subsequently emerged to the gas cell chamber. The background pressure in the gas cell chamber $P_{\text {bg }}$ was adjusted by changing the rotational speed of the utilized screw pump GXS 450/4200 (890 l/s) from Edwards Vacuum and by partially closing the valve between the gas cell chamber and the screw pump. Within the PLIF experiments presented in this paper, the fluorescence light was collected by an objective lens of an intensified CCD (ICCD) camera, installed at a distance of $10 \mathrm{~cm}$ from the jet. A 5.7-cm-diameter objective lens of the camera resulted in a solid angle efficiency of $2 \%$ of $4 \pi$. 


\section{Laser system}

The PLIF experiments were performed using laser radiation of different bandwidths. The broadband dye laser (CREDO, Sirah Lasertechnik GmbH) was operated with a dye solution of DCM in dimethyl sulfoxide (DMSO) and pumped with the second harmonic of a Nd:YAG laser (INNOSLAB, EdgeWave $\mathrm{GmbH}$ ) at $532 \mathrm{~nm}$ to generate tunable laser light at around $654.98 \mathrm{~nm}$ with a bandwidth of about 2.50(6) $\mathrm{GHz}$ [48]. This light was then frequency doubled in a nonlinear barium borate (BBO) crystal to finally obtain the laser light at $327.49 \mathrm{~nm}$ (wavelength in vacuum), used for excitation. The relative density profiles of supersonic jets were obtained by evaluating the intensity of the fluorescent light recorded on each pixel of the camera with the PLIF spectroscopy performed using the broadband laser. In order to perform spectroscopy studies and thus to extract the velocity and temperature profiles, a laser with a reduced bandwidth was used. The narrow band laser radiation was obtained by amplifying a continuous wave (cw) single-mode diode laser (TOPTICA Photonics AG) at $654.98 \mathrm{~nm}$ in a two-stage pulsed dye amplifier pumped with the second harmonic of the Nd:YAG laser. The amplified pulsed radiation was then frequency doubled, resulting in a spectral bandwidth of the UV light of about $70 \mathrm{MHz}$ stemming from a laser pulse with a Gaussian time profile of $\sim 7 \mathrm{~ns}$ full width at half maximum [23].

To probe a larger area of the jet in the transverse direction, the laser beam was reshaped in the horizontal axis to a light sheet of $40 \mathrm{~mm}$ width using a cylindrical telescope (transverse direction in Fig. 2). The latter consisted of a set of plano-concave and plano-convex lenses, with a limitation on the maximum length of the collimated laser sheet given by the $2 \mathrm{in}$. diameter of the last mirror installed just in front of the gas cell chamber. A divergent laser sheet should be avoided when performing spectroscopy as it causes an additional linewidth broadening [23]. The laser beam was also focused in the vertical axis to a thickness of about $1 \mathrm{~mm}$ by a spherical lens with a $1 \mathrm{~m}$ focal length to restrict the laser-gas jet intersection region to a thin slice of the gas jet, assuring a local characterization of the jet parameters. A helium-neon laser was used for the alignment of the laser sheet with the gas jet along its central line. In the case of the PLIF experiments performed in the longitudinal direction, the spherical lens, cylindrical telescope, and mirror $M 1$ of the transverse direction were removed from the laser beam path. For the longitudinal direction between the laser beam and the jet, a cylindrical telescope consisting of plano-concave and plano-convex cylindrical lenses was used for the laser beam reshaping and collimation in the horizontal and vertical axes independently. The typical values of the used laser energy fluence were $\Phi \sim 10 \mathrm{pJ} / \mathrm{mm}^{2}$ for the narrow band laser, while for the broadband laser $\Phi$ ranged from 0.25 to $1 \mu \mathrm{J} / \mathrm{mm}^{2}$ for the longitudinal direction and $\Phi \sim 20 \mathrm{~nJ} / \mathrm{mm}^{2}$ for the transverse direction.

\section{B. Design and manufacturing of de Laval nozzles}

A de Laval nozzle is a convergent-divergent contoured nozzle used to accelerate gas flows to supersonic velocities. The acceleration is achieved by transformation of the gas thermal energy into kinetic energy. Acceleration of a subsonic flow $(M<1)$ up to a velocity of the local speed of sound takes place in the convergent part of the nozzle with a subsequent acceleration of a $M>1$ flow in the divergent part.

In total, we investigated four different nozzles, for two of them numerical calculations were performed using the computational fluid dynamics (CFD) module of the COMSOL Multiphysics software with an aim to calculate the flow properties of the jets as they emerged to the gas cell chamber. The CFD module is a numerical simulation tool for solving full Navier-Stokes equations [49]. The inner contour of nozzle 1 was calculated using another software package called Nozzle 3.7 that is only able to model an inviscid, adiabatic, and isentropic flow of a calorically perfect gas, and thus, a limited performance with respect to the uniformity of the jet was expected. However, fast calculations of inner contours are feasible with the Nozzle 3.7 software package. The inner contour of nozzle 2 was optimized using the CFD module. The calculated properties of the gas jet formed by nozzle 1 , whose inner contour was imported to the CFD module, and nozzle 2 are presented in Fig. 3 for adjusted values of the background pressure $P_{\mathrm{bg}}$ that minimizes density fluctuations along the central jet line. Enlargements of density profiles are shown in the insets. The numerical calculations were performed for a gas flow of argon, $P_{0}=300 \mathrm{mbar}, T_{0}=300 \mathrm{~K}$, a nozzle throat of a diameter $d^{*}=1 \mathrm{~mm}$, and a uniform gas inflow at the nozzle inlet. Additionally, a new nozzle design was calculated using the same code as for nozzle 1 and was manufactured in two versions (nozzle 3 and nozzle 4) with different surface finishing, resulting in different roughness $R_{a}$, defined as the average deviation of the roughness profile with respect to the mean value within the evaluation length. An overview of the four investigated nozzles is given in Table I.

The remaining gas density fluctuations, calculated as $\Xi=\left(\max _{\rho}-\min _{\rho}\right) / \operatorname{mid}_{\rho} \times 100 \%$ are $48 \%$ for nozzle 1 [see the inset of the density variations along the central jet line in Fig. 3(a)] and have a period of fluctuations $\Lambda \approx 17 \mathrm{~mm}$. The pressure ratio between the gas cell and the gas cell chamber at which the minimized density fluctuation was obtained is $P_{0} / P_{\mathrm{bg}}=265$. The variations of the jet parameters along the central jet line are, however, still present with the maximum value of the Mach number $M=5.3$. Moreover, limitations of the software used to model the inner contour of nozzle 1 manifest themselves in an inhomogeneous color map of the Mach number [see Fig. 3(a)]. An estimation of the period $\Lambda$ can be done in the following way. Small disturbances in supersonic flow propagate along characteristics or Mach lines which form 

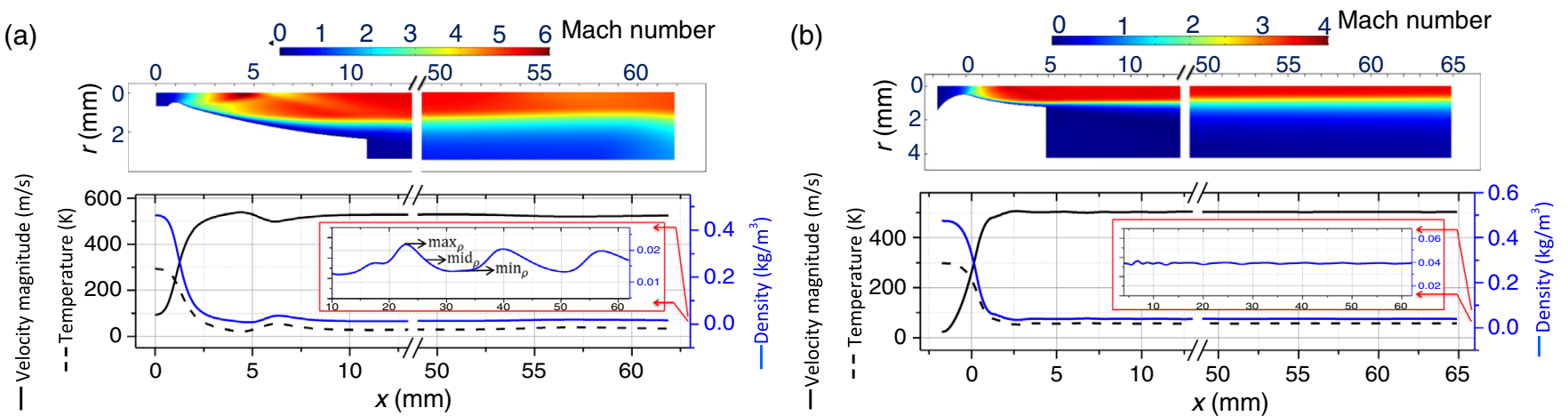

FIG. 3. Numerical calculations using the CFD module of COMSOL of the flow parameters for nozzle 1 and nozzle 2 . The color bar indicates the Mach number of the gas flow. The velocity, temperature, and density along the central jet line are shown. $P_{0}=300 \mathrm{mbar}, T_{0}=300 \mathrm{~K}$, and $d^{*}=1 \mathrm{~mm}$. (a) Nozzle 1: $P_{\mathrm{bg}}=1.13 \mathrm{mbar}$, the nozzle exit is at $x_{\text {exit }}=10.8 \mathrm{~mm}$. (b) Nozzle 2 : $P_{\mathrm{bg}}=4.6$ mbar, $x_{\text {exit }}=4.9 \mathrm{~mm}$.

an angle $\beta=\arcsin (1 / M)$ with the flow direction [50]. The period $\Lambda$ is an axial distance needed for a disturbance to travel from the axis to a side of the jet and back. Hence, by taking into account the diameter of the supersonic jet at the nozzle exit $d_{\text {jet }}$, the period $\Lambda$ can be written as

$$
\Lambda=d_{\text {jet }} \sqrt{M^{2}-1}
$$

where

$$
\left(\frac{d_{\text {jet }}}{d^{*}}\right)^{2}=\left[\frac{2}{\gamma+1}\left(1+\frac{\gamma-1}{2} M^{2}\right)\right]^{(\gamma+1) /[2(\gamma-1)]} \frac{1}{M}
$$

Here, $\gamma$ is the adiabatic index (for monoatomic gases, as the noble gases, $\gamma=5 / 3$ ). Equation (1) gives $\Lambda=17.7 \mathrm{~mm}$ for $M=5.3$ and $d_{\text {jet }}=3.4 \mathrm{~mm}$ [Eq. (2), $d^{*}=1 \mathrm{~mm}$, which is in good agreement with the period of $17 \mathrm{~mm}$ obtained with the CFD module for nozzle 1 (see Table I). For nozzle 2, the pressure ratio at which the minimized density fluctuations were obtained is $P_{0} / P_{\mathrm{bg}}=65$. The remaining density fluctuations are $\Xi=4 \%$ and have a period $\Lambda=8 \mathrm{~mm}$ for $x$ coordinates from 15 to $60 \mathrm{~mm}$. The numerical calculations showed that a jet of $M=3.6$ is formed. An estimation of the period $\Lambda$ using Eq. (1) gives
$\Lambda=7.3 \mathrm{~mm}$ for $M=3.6$ and $d_{\text {jet }}=2.1 \mathrm{~mm}$, which is in good agreement with the period of $8 \mathrm{~mm}$ obtained with the CFD module for nozzle 2 . The diameter $d_{\text {jet }}$ is smaller than the nozzle exit diameter $d_{\text {exit }}$ due to the presence of the boundary layer.

The nozzles were manufactured out of stainless steel by two different techniques: nozzle 1 , nozzle 3 , and nozzle 4 by high-precision turning in a computer numerical control machine (CNC turning) with a different finishing of the inner contour, resulting in a different surface roughness $R_{a}$, and nozzle 2 by electrical discharge machining (EDM). A deviation tolerance of $5 \mu \mathrm{m}$, i.e., a final machined surface is within $5 \mu \mathrm{m}$ from the nominal surface, and a small surface roughness $R_{a}<1 \mu \mathrm{m}$ were desired objectives for the inner contour. For nozzle 3 and nozzle 4 these close tolerances and, moreover, large "depth-to-diameter" ratio of the nozzle supersonic part limited the possible machining techniques of the inner contour. In order to reach the envisaged requirements, a precision turning operation on a CNC controlled machine (Spinner SB-CNC) was performed using just one cutting tool to avoid possible discontinuities in the inner contour. Lacking commercially available suitable cutting tools, a special tool was designed (see the inset in Fig. 4), subsequently fabricated out of tungsten carbide, and coated with titanium aluminium

TABLE I. An overview of the designed and manufactured nozzles. The following parameters are mentioned: the software used to calculate the inner contours, the ratio between the nozzle exit and throat diameters $d_{\text {exit }} / d^{*}$, the pressure ratio between the gas cell and the gas cell chamber $P_{0} / P_{\mathrm{bg}}$, the remaining density fluctuations $\Xi$ and their periodicity $\Lambda$, the Mach number $M$, the manufacturing technique, and the surface roughness of the nozzle inner contour $R_{a}$. $d^{*}=1 \mathrm{~mm}, P_{0}=300$ mbar, and $T_{0}=300 \mathrm{~K}$ for all cases.

\begin{tabular}{lcccccccc}
\hline \hline Nozzles & Software & $d_{\mathrm{exit}} / d^{*}$ & $P_{0} / P_{\mathrm{bg}}$ & $\Xi(\%)$ & $\Lambda(\mathrm{mm})$ & $M$ & Manufacturing technique & $R_{a}(\mu \mathrm{m})$ \\
\hline Nozzle 1 & Nozzle 3.7 & 4.72 & 265 & 48 & 17 & $5.3^{\mathrm{a}}$ & CNC turning & $\ldots$ \\
Nozzle 2 & CFD module & 2.48 & 65 & 4 & 8 & 3.6 & EDM & $\ldots$ \\
Nozzle 3 & Nozzle 3.7 & 8.66 & $3151^{\mathrm{b}}$ & $\ldots$ & $\cdots$ & $8.5^{\mathrm{b}}$ & CNC turning & 0.8 \\
Nozzle 4 & Nozzle 3.7 & 8.66 & $3151^{\mathrm{b}}$ & $\cdots$ & $\cdots$ & $8.5^{\mathrm{b}}$ & CNC turning & 0.4 \\
\hline \hline
\end{tabular}

${ }^{\mathrm{a}}$ The maximum value of the varying Mach number of nozzle 1.

${ }^{\mathrm{b}}$ The projected value of the Mach number of nozzle 3 and nozzle 4 and the pressure ratio $P_{0} / P_{\mathrm{bg}}$ as calculated using Eq. (14). 


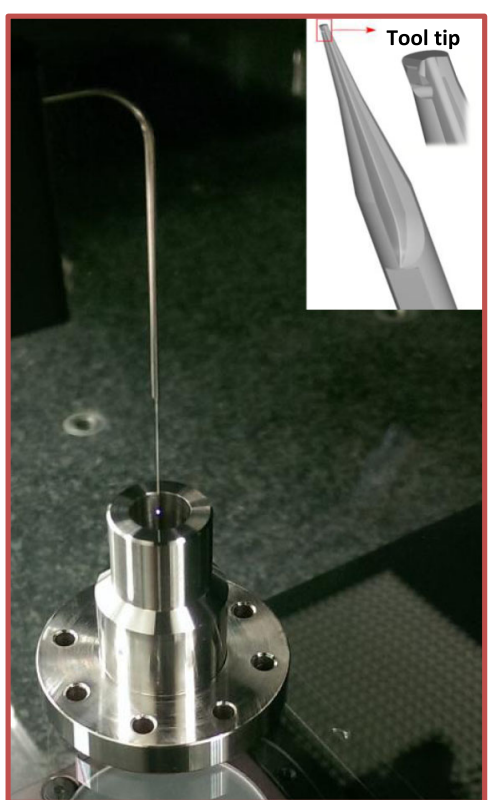

FIG. 4. Nozzle 4 installed in a high-end precision coordinate measuring machine Werth VideoCheck HA in a combination with a fiber probe for measurement of the nozzle inner contour (see Sec. V). A design geometry of a custom-fabricated internal turning tool is shown in the inset.

nitride (TiAlN). The tool has a minimum turning diameter of $1 \mathrm{~mm}$ and a total length of $53 \mathrm{~mm}$, which is sufficient to finish the inner contour from the nozzle throat till the exit diameter in a single and continues cut. Hand polishing of the inner surface with $5 \mu \mathrm{m}$ silicon carbide lapping foil allowed further reduction of the surface roughness $R_{a}$ of nozzle 4 to $0.4 \mu \mathrm{m}$ compared to the roughness $R_{a}$ of $0.8 \mu \mathrm{m}$ of nozzle 3 . Stainless steel was initially chosen as a nozzle material for its heat expansion coefficient that is sufficiently small to minimize modifications of the inner contour of the nozzle due to the cryogenic temperatures in the core of the high Mach number jet and for its high wear and corrosion resistance. However, as we discuss in Sec. IV B, only a minor drop of the temperature of the nozzle inner contour of nozzle 3 was measured in working conditions.

\section{Atomic PLIF spectroscopy}

The copper atoms were excited from the $4 s^{2} S_{1 / 2}$ ground state to the $4 p^{2} P_{1 / 2}$ excited state (see Fig. 5) by broadband or narrow band laser light at $327.40 \mathrm{~nm}$. The fluorescence light at $578.21 \mathrm{~nm}$, emitted upon radiative deexcitation to a $4 s^{2}{ }^{2} D_{3 / 2}$ metastable state with a branching ratio of $1.4 \%$ [51], was subsequently recorded by an iStar ICCD camera (ANDOR-DH334T). The accumulated images were transferred to a computer for data processing and storage. In this way, the collected fluorescence light could be used for a detailed pixel-by-pixel analysis of the jet structure. The main features of the camera were a photocathode quantum

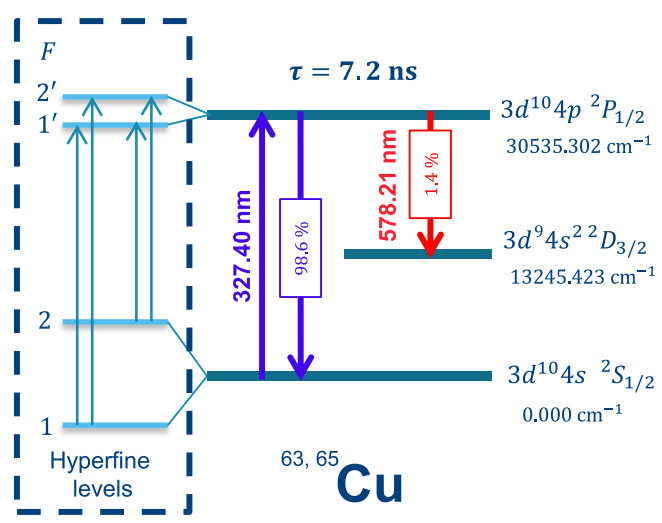

FIG. 5. Atomic levels and their hyperfine splitting (not to scale) in the stable ${ }^{63,65} \mathrm{Cu}$ isotopes.

efficiency of $45 \%$ at the fluorescence wavelength, a $13 \mu \mathrm{m}^{2}$ pixel size (with a resulting spatial resolution in the in-gasjet visualization plane of $0.2 \mathrm{~mm}$ ), and the possibility to synchronize the trigger of the photocathode with the fluorescence detection, even allowing for delayed acquisition of the fluorescence light. The larger pixel size was important to enhance the sensitivity but, on the other hand, it limited the spatial resolution. The camera sensor was cooled to a temperature of $-30{ }^{\circ} \mathrm{C}$ in order to reduce the dark current and associated shot noise. The images were acquired after accumulation of $\sim 3 \times 10^{5}$ laser pulses, with each of them triggering the photocathode to open for $50 \mathrm{~ns}$, resulting in a full time overlap between the laser pulse $(\sim 7 \mathrm{~ns}$ width) and the photocathode acquisition time window. The collection of light over a considerable number of laser pulses, which was essential to obtain an adequate signal-to-noise ratio (SNR), resulted in time-averaged jet characteristics. Scattered light from the laser and the light from the direct deexcitation of the excited state to the ground state were attenuated by long-pass optical filters installed in front of the camera. Acquired fluorescence images were processed in order to subtract the remaining background light and/or to correct for a nonuniform light intensity along the laser sheet, if it was required.

To estimate the total flux of copper atoms in the gas jet, we first performed in-gas-cell resonance laser ionization with broadband lasers. The total copper ion current measured at the detection station with the Faraday cup was $\sim 160 \mathrm{pA}$, which corresponds to $1 \times 10^{9}$ copper ions per second. As the efficiency of the ionization step was less than $100 \%$, and thus more copper atoms were expected to contribute to the fluorescence signal than to the ion current, this led to an estimated lower limit of $1 \times 10^{9}$ copper atoms at the $4 p{ }^{2} P_{1 / 2}$ excited state, which can contribute to the fluorescence signal. However, due to the $1.4 \%$ branching to the metastable state, only $1.4 \times 10^{7}$ atoms emitted fluorescence light upon deexcitation to the metastable state, whereas the rest decayed following the transition back to the ground state. 
An efficiency of PLIF spectroscopy can be estimated as

$$
Y=\eta_{1} \times \eta_{2} \times \eta_{3} \times \eta_{4} \times X,
$$

where $X$ is the number of the copper atoms decaying to the metastable state and $Y$ is the number of the photoelectrons emitted by the camera photocathode. $\eta_{1}$ is a correction factor for the solid angle efficiency of the camera's objective lens $\left(\eta_{1}=2 \%\right)$. The laser sheet in the transverse direction interacted only with a thin layer of the gas jet and thus only with a fraction of the atoms, which led to the jet area correction factor $\eta_{2}=42 \%$ for a jet diameter of $3 \mathrm{~mm}$ at $M=5$, with a nozzle throat diameter $d^{*}=1 \mathrm{~mm}$ and a laser sheet thickness of $1 \mathrm{~mm}$. A complete temporal overlap between the pulsed laser radiation and the copper atoms in the continuous high-velocity flow was assumed in this estimation $\left(\eta_{3}=100 \%\right)$. The long-pass optical filters, the objective lens, and the photocathode spectral sensitivity significantly reduce the scattered pump light and the fluorescence from the direct deexcitation to the ground state, while providing a transmission $\eta_{4}=25 \%$ at the fluorescence wavelength.

Following these estimations, we expect to have about $2.9 \times 10^{4}$ photoelectrons emitted by the photocathode for the $1.4 \times 10^{7}$ copper atoms decaying to the metastable state with the efficiency of the PLIF spectroscopy of $\sim 0.2 \%$.

The photoelectrons, emitted by the photocathode, were accelerated and amplified in the image intensifier MCP and hit a phosphor screen. The photons subsequently emitted from the phosphor screen reached a 1024 pixel $\times$ 1024 pixel CCD sensor with the jet image occupying only about $0.5 \%$ of the sensor area. The small pixel area was the result of a trade-off between the SNR and the required spatial resolution. Accordingly, a signal $S \sim$ 830 counts $/ \mathrm{pixel} / \mathrm{s}$ was expected at the maximum MCP gain of $\times 150$ with a corresponding signal shot noise of about 29 (i.e., $\sqrt{S}$ ). The camera readout noise was found to be $19.8 e^{-} /$pixel (resulting in 4 counts/pixel) and the dark current was reduced to $\sim 0.25 e^{-} / \mathrm{pixel} / \mathrm{s}$ (i.e., 0.05 counts/pixel/s). A signal $S$ of about $830 \times 30$ counts/pixel was reached by accumulating fluorescent light following $\sim 3 \times 10^{5}$ laser pulses (at a pulse repetition rate of $10 \mathrm{kHz}$ ) resulting in a total accumulation time of $30 \mathrm{~s}$. Because of the small contribution of the readout noise and the shot noise of dark current, the SNR $\sim \sqrt{S}$ and thus results in a value of about 160 .

Because of the residual Doppler broadening, only a fraction of the copper atoms was excited at each laser frequency in the case of spectroscopy measurements with the narrow band laser, and thus the SNR was reduced. The jet images were taken with the camera for a set of laser frequencies covering about $7.5 \mathrm{GHz}$ with a frequency step of $\sim 100 \mathrm{MHz}$. These images were subsequently processed to extract the local variations of the light intensity collected in each (or a group of) pixels as a function of the laser frequency. In this way, the hyperfine spectra of copper from different jet regions were obtained and variations of the local jet characteristics, such as linewidth $\Delta_{V}$, Doppler shift $\Delta \nu$, velocity $v$, temperature $T$, and Mach number $M$, were extracted, as we discuss in the following section.

\section{Spectrum analysis}

The copper atoms move at high velocities along with the argon in the jet, leading to a Doppler shift (relative to that with the atoms at rest) of the atomic transition frequencies $\nu_{0}$ for the case of longitudinal excitation. The Doppler shift can be written as

$$
\Delta \nu=\nu_{0} \frac{v}{c},
$$

where $c$ is the speed of light and $v$ is the velocity of the atoms seeded into the argon flow. $\nu_{0}$ is the frequency of the electron transition for the atoms at rest or moving perpendicular to the observation axis for the transition from the hyperfine level $F=1$ of the $4 s^{2} S_{1 / 2}$ ground state to the hyperfine level $F=2^{\prime}$ of the $4 p^{2} P_{1 / 2}$ excited state in ${ }^{63} \mathrm{Cu}$ (see Fig. 5), which was measured in an atomic beam unit (ABU) prior to the PLIF experiments. $\Delta \nu$ is positive for a collinear configuration between the laser beam and the gas jet. In this way, the local velocity component of the copper atoms in the direction of the laser beam was determined from the measured Doppler shift $\Delta \nu$.

Extracting the gas temperature from the spectral linewidth is a less trivial task due to the presence of a number of linewidth broadening mechanisms. The Voigt profile resulting from the PLIF spectroscopy measurements is a convolution of Gaussian and Lorentzian profiles with a FWHM given by [52]

$$
\Delta_{V}=0.5346 \Gamma+\sqrt{0.2166 \Gamma^{2}+\Delta_{G}^{2}},
$$

where $\Delta_{G}$ and $\Gamma$ are the linewidths (FWHM) associated to the Gaussian and Lorentzian distributions, respectively.

The Doppler effect on the Maxwell-Boltzmann velocity distribution due to the temperature of the atoms and the divergence of the atomic copper beam in addition to the laser bandwidth contribute to the Gaussian linewidth [23]:

$$
\Delta_{G}=\sqrt{\left(\Delta \nu_{\text {Doppler }}\right)^{2}+\left(\Delta \nu_{\text {laser }}\right)^{2}}
$$

where $\Delta \nu_{\text {laser }}$ is the laser bandwidth. The FWHM of the Maxwell-Boltzmann velocity distribution can be written as

$$
\Delta \nu_{\text {Doppler }}=7.16 \times 10^{-7} \times \nu_{0} \times \sqrt{T / A},
$$

where $T$ is the absolute temperature in kelvin in the region of intersection of the atomic beam with the laser beam and $A$ is the atomic mass number. The divergence of the atomic 
beam has a small contribution to the total Doppler width for the case of longitudinal direction since only the velocity components $v \cos \theta$ contribute to the additional linewidth broadening. The contribution of the beam divergence to the total linewidth can be written as

$$
\Delta \nu_{\mathrm{ax}}=\nu_{0} \times v \times(1-\cos \theta) / c,
$$

which results in about $6 \mathrm{MHz}$ for the studied transition in copper, assuming $v=550 \mathrm{~m} / \mathrm{s}$ and a small beam divergence angle $\theta=5^{\circ}$. This contribution to the linewidth is well below the temperature-associated broadening, as obtained during the in-gas-jet experiments (see Secs. III and IV B), and thus can be neglected.

The decay of the excited state, collisions with the buffer gas, and the laser power broadenings contribute to the Lorentzian linewidth:

$$
\Gamma=\Gamma_{\text {nat }}+\Gamma_{\text {coll }}+\Gamma_{\text {power }}
$$

where $\Gamma_{\text {nat }}$ is a natural linewidth of the excited state, and $\Gamma_{\text {coll }}$ and $\Gamma_{\text {power }}$ are collision and power linewidths, respectively. The $4 p^{2} P_{1 / 2}$ excited state in copper has a natural linewidth of $22 \mathrm{MHz}$ corresponding to the Einstein coefficient for spontaneous emission, $A_{01}=1.361 \times 10^{8} \mathrm{~s}^{-1}$ [51]. An excess of the laser power results in a linewidth broadening that can potentially cause a masking of the hyperfine structure and thus hamper the precise determination of the Gaussian linewidth. For this reason, the PLIF spectroscopy was performed at sufficiently small values of the laser energy fluence $\Phi$ at which the presence of power broadening could be neglected. Whereas the collision broadening coefficient $\gamma_{\text {coll }}^{0}$ for the atomic transition $3 d^{10} 4 s^{1} \rightarrow 3 d^{10} 4 p$ used in these studies has not been experimentally determined (to the best of our knowledge), such a coefficient has been measured for the atomic transition $3 d^{10} 4 s^{1} \rightarrow 3 d^{9} 4 s 4 p$ at room temperature using argon as a buffer gas and found to be $5.4 \mathrm{MHz} / \mathrm{mbar}$ [53]. Typical collision broadening coefficients do not exceed $10 \mathrm{MHz} / \mathrm{mbar}$ [54] and, moreover, are reduced at low temperatures [55]. In a low-density and cold argon jet one can therefore expect a minor contribution of the collision broadening to the Lorentzian linewidth. Alongside the broadening of the spectral lines, collisions of the copper atoms with the buffer gas cause the spectral lines to shift. The typical collision shift coefficients are a fraction of the values of the corresponding broadening coefficients, which can be neglected for the in-gas-jet experiments. Following this line of reasoning, the Lorentzian linewidth was fixed to the natural linewidth $\Gamma_{\text {nat }}$.

All hyperfine spectra of copper were analyzed using a PYTHON package, the statistical analysis toolbox for laser spectroscopy (SATLAS) [56]. The spectra were fitted with the nonlinear least-squares algorithm, using a Voigt line profile. The Gaussian linewidth $\Delta_{G}$ was set to be equal for all peaks of the hyperfine structure of ${ }^{63} \mathrm{Cu}$ and
${ }^{65} \mathrm{Cu}$ isotopes; the same was set for the Lorentzian linewidth $\Gamma$. The hyperfine splitting of the electronic ground and excited states [57], the natural abundance of the stable copper isotopes, and the relative amplitudes of the peaks of the same isotope as given by the Racah coefficient were fixed in the fittings. The local velocity $v$ was extracted from the measured Doppler shift $\Delta \nu$ by using Eq. (4). The measured Voigt linewidth $\Delta_{V}$ was used to deduce $\Delta \nu_{\text {Doppler }}$, from which the local temperature $T$ was extracted using Eq. (7).

\section{VALIDATION OF ATOMIC PLIF SPECTROSCOPY USING SUPERSONIC FREE JETS}

Supersonic free jets, formed by the gas emitted from the exit section of a convergent nozzle to the ambient medium at a lower pressure, have been extensively studied over the past few decades $[31,33,34,42,43,58-63]$ with the research in this area stimulated by both the fundamental fluid mechanics and practical applications of such gas jets (e.g., rocket exhaust plumes, vehicle control jets, and jets emanating from young stars). The main details of a supersonic free jet structure are shown in Fig. 6, and a detailed description can be found elsewhere (see, e.g., Ref. [50]).

Studies of the geometry of the highly underexpanded supersonic jets have been performed both analytically and experimentally $[58,59]$. Those studies showed that the jet parameters within the "zone of silence" (see Fig. 6) can be characterized as follows.

(1) The flow streamlines appear to originate from a spherical source, placed at a distance $x_{0}$ downstream of the nozzle exit.

(2) The density $\rho$ decreases along the streamline proportionally to the inverse square of the distance $R$ from the source $\left(\rho \propto 1 / R^{2}\right)$.

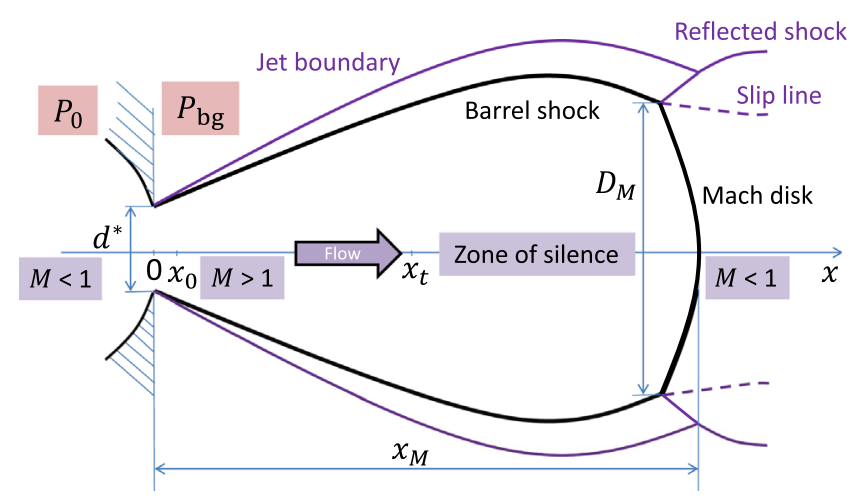

FIG. 6. Structure details of an axisymmetric (with revolution symmetry around the $x$ axis) supersonic free jet. The nozzle exit is located at $x=0$. The nozzle exit diameter $d^{*}$, the coordinate $x_{M}$, and diameter $D_{M}$ of the Mach disk are indicated. 
(3) An expression for the Mach number variations (for $M>5.5)$ as a function of the distance $x$ from the nozzle exit is given by

$$
M=C_{1}\left(\frac{x-x_{0}}{d^{*}}\right)^{\gamma-1}-\frac{1}{2} \frac{\left(\frac{\gamma+1}{\gamma-1}\right)}{C_{1}\left(\frac{x-x_{0}}{d^{*}}\right)^{\gamma-1}},
$$

where $d^{*}$ is the exit diameter of the supersonic free jet nozzle. $C_{1}$ and $x_{0}$ are constants that for $\gamma=5 / 3$ have the values of $C_{1}=3.26$ and $x_{0} / d^{*}=0.075$. A nozzle with an exit diameter of $1 \mathrm{~mm}$ was utilized at the IGLIS laboratory and thus $x_{0}=0.075 \mathrm{~mm}$, which is less than the spatial resolution of $0.2 \mathrm{~mm}$ achieved with our experimental setup. In this way, we expect the observed streamlines of the supersonic free jets to originate from a spherical source located at the nozzle exit position.

A fit of the Mach number along the central jet line for $M<5.5$ is given by $[64,65]$

$M=\tilde{x}^{(\gamma-1)}\left(C_{2}+\frac{C_{3}}{\tilde{x}}+\frac{C_{4}}{\tilde{x}^{2}}+\frac{C_{5}}{\tilde{x}^{3}}\right), \quad$ for $\tilde{x}>0.5$,

and

$$
M=1+C_{6} \tilde{x}^{2}+C_{7} \tilde{x}^{3}, \quad \text { for } 0<\tilde{x}<1,
$$

where $\tilde{x}=x / d^{*}, C_{2}=3.232, C_{3}=-0.7563, C_{4}=0.3937$, $C_{5}=-0.0729, C_{6}=3.337$, and $C_{7}=-1.541$.

In the adiabatic flow of a perfect gas, the local flow temperature $T$ and pressure $P$ can be described as follows [66]:

$$
\begin{gathered}
T=\frac{T_{0}}{1+\frac{\gamma-1}{2} M^{2}}, \\
P=\frac{P_{0}}{\left(1+\frac{\gamma-1}{2} M^{2}\right)^{\gamma /(\gamma-1)}} .
\end{gathered}
$$

In addition,

$$
v=M a=M \sqrt{\frac{\gamma k T}{m}},
$$

where $v$ is the flow velocity, $a$ is the speed of sound, $k$ is the Boltzmann constant, and $m$ is the mass of the gas atom. By introducing Eqs. (10)-(12) to Eqs. (13) and (15), the expressions for the temperature $T$ and the velocity $v$ as a function of the distance $x$ from the nozzle exit can be derived.

The density and temperature, and thus the collision rate, drop quickly in the expanding flow of supersonic free jets, which can be divided into two regions with different flow regimes. In an approximate treatment of the transition between these two regimes, referred to as the "sudden freeze model," it is assumed that the transition occurs through a spherical surface confined by a barrel shock, with the continuous regime dominated by the collisions upstream of the surface and the free molecular regime with rare molecular collisions downstream of the surface. The axial velocity and the Mach number get frozen at the transition to the free molecular regime. The terminal Mach number $M_{t}$ [67], which can be reached in the expanding flow of the supersonic free jets at a distance $x_{t}$ (see Fig. 6) from the nozzle exit, is given by

$$
M_{t}=3.32\left(P_{0} d^{*}\right)^{0.4}
$$

and

$$
\frac{x_{t}}{d^{*}}=\left(\frac{M_{t}}{3.26}\right)^{1.5}
$$

where $P_{0}$ is in mbar and $x$ and $d^{*}$ are in mm.

The distance $x_{M}$ of the Mach disk, or the normal shock wave, from the nozzle exit has been found to follow the equation $[59,68]$

$$
\frac{x_{M}}{d^{*}}=0.67 \sqrt{\frac{P_{0}}{P_{\mathrm{bg}}}},
$$

which holds for $15 \leq P_{0} / P_{\mathrm{bg}} \leq 17000$ and is independent of $\gamma$.

The aforementioned expressions were used to verify the correct characterization of the thermodynamic and fluid dynamic parameters of the gas jets by atomic PLIF spectroscopy.

Firstly, PLIF spectroscopy was performed in the transverse direction using the broadband laser. In this case, the measured fluorescence signal was proportional to the local density of the copper atoms seeded into the argon flow. Figure 7 shows the coordinates of the Mach disks $x_{M}$ that were extracted as $x_{M}=x_{\min }+\left(x_{\text {const }}-x_{\min }\right) / 2$ for different pressure ratios $P_{0} / P_{\mathrm{bg}}$. The coordinates showed good agreement with the earlier experimental results (the black line in Fig. 7). The obtained images of supersonic free jets are shown in the insets. The background associated with the remaining scattered laser light and the dark current has been subtracted by acquiring images when the filament heating was switched off. The supersonic free jets had a nonaxisymmetric structure, which is explained by a nonaxisymmetric distribution of copper atoms at the gas cell exit due to a bent flow inside it (see Fig. 2).

Additionally, a measurement using the broadband laser was performed in the longitudinal direction. The drop in the fluorescence signal, proportional to the density of the copper atoms, is shown in Fig. 8 as a function of the $x$ coordinate along the central jet line. No significant 


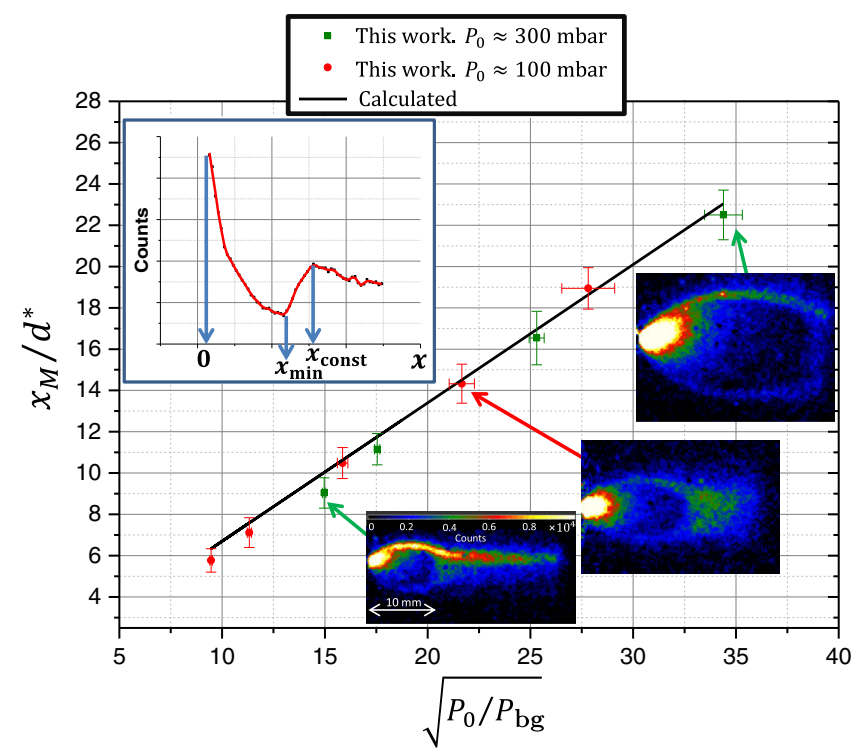

FIG. 7. Distance $x_{M}$ of the Mach disk from the nozzle exit at different values $\sqrt{P_{0} / P_{\mathrm{bg}}} \cdot d^{*}=1 \mathrm{~mm}$. The green and red symbols correspond to $P_{0} \approx 300 \mathrm{mbar}$ and $P_{0} \approx 100 \mathrm{mbar}$ as measured with the PLIF spectroscopy, respectively. The black line shows the outcome after using Eq. (18) [59,68]. The color code of the images of supersonic free jets indicates the number of counts collected at a pixel due to the fluorescence signal (white color corresponds to the most intense fluorescence signal). The inset shows the observed fluorescence signal as a function of the distance from the exit hole recorded for the central jet line. The positions of $x_{\min }$ and $x_{\text {const }}$, used to determine $x_{M}$ as explained in the text, are indicated.

deviation from the $1 / R^{2}$ trend was observed even for $x$ coordinates close to the nozzle exit. The background associated with the remaining scattered laser light and the dark current has been subtracted.

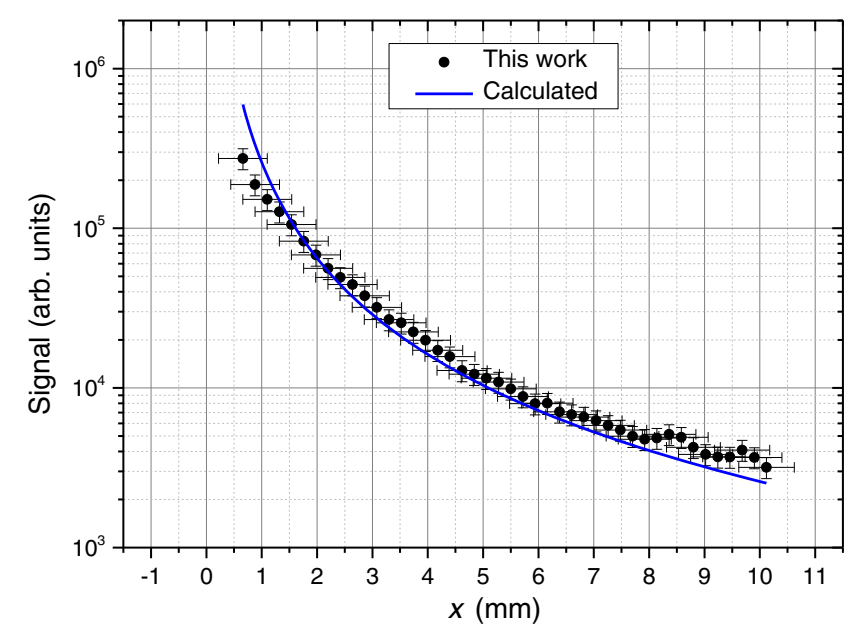

FIG. 8. Fluorescence signal drop along the central jet line from the PLIF measurements (black symbols). $P_{0}=360 \mathrm{mbar}$, $P_{\mathrm{bg}}=1.5$ mbar. The expected drop of the density following a $1 / R^{2}$ trend is shown with a blue curve [50].
In the expanding flow of the supersonic free jet, the local argon pressure reaches values above the argon liquid-vapor equilibrium for the local temperature, so the condensation of argon can take place. However, due to the fast density drop in the expanding gas flow, there is only a small region in which the argon densities are still sufficiently high for the argon condensate to be formed. In either way, the PLIF spectroscopy was performed with the fluorescence light emitted by the copper atoms, and the drop in fluorescence signal showed no deviation from the expected $1 / R^{2}$ trend, caused by the supersonic free jet expansion. Thus, we cannot confirm or neglect the presence of the argon condensate in the jet medium. On the other hand, we can conclude, within our experimental sensitivity, that copper atoms did not form dimers or clusters with argon. However, the formation of copper-argon molecular ions was observed during in-gas-jet laser ionization experiments at the LISOL facility [69], which caused a reduction of the total production efficiency.

Secondly, the transitions from the hyperfine level $F=1$ of the ground state $4 s^{2} S_{1 / 2}$ to the hyperfine levels $F=1^{\prime}$ and $F=2^{\prime}$ of the excited state $4 p^{2} P_{1 / 2}$ (see Fig. 5) were scanned with the narrow band laser in transverse direction. Owing to the narrow bandwidth of the laser, and thus to the sensitivity to the different components of the velocity vectors in the direction of the laser beam, the visualization of different flow streamlines in the expanding flow of the supersonic free jet was possible (Fig. 9). The laser frequency was in resonance with the $v \sin \theta$ component of the velocity vectors, as shown in Fig. 9. The figure shows two images with different settings of the laser frequency: on resonance on the main peak $A$ of ${ }^{63} \mathrm{Cu}(\Delta \nu=0 \mathrm{GHz})$ and off resonance at $\Delta \nu=+0.5 \mathrm{GHz}$. The on-resonance image shows a horizontal jet streamline, indicated with the arrow (A) and corresponding to the peak $A$ of ${ }^{63} \mathrm{Cu}$ atoms as in the transverse geometry; no Doppler shift is present. The offresonance image shows two distinct jet streamlines. The one moving away from the laser is mainly due to the peak $A$ of ${ }^{63} \mathrm{Cu}$ atoms whose resonance has been redshifted while the jet streamline moving towards the laser stems from the peak $B$ of ${ }^{65} \mathrm{Cu}$ atoms with a blueshifted resonance.

Finally, PLIF spectroscopy was performed using the narrow band laser beam in longitudinal direction at $P_{0}=$ 355 mbar and $P_{\mathrm{bg}}=1.3 \mathrm{mbar}$. To ensure the absence of power broadening, the narrow band scan was performed with a laser energy fluence $\Phi \sim 10 \mathrm{pJ} / \mathrm{mm}^{2}$. The analysis of the obtained data was performed as described in Sec. II D, resulting in the extraction of the local characteristics along the central jet line, as shown in Fig. 10. The Mach number $M$ was deduced from the obtained velocity and temperature values using Eq. (15) (here, $v$ is the velocity of seeded copper atoms). Extra attention had to be paid to the analysis of the hyperfine spectra for the regions close to the nozzle exit due to contributions of the beam divergence, collision broadening, and collision shift, which 

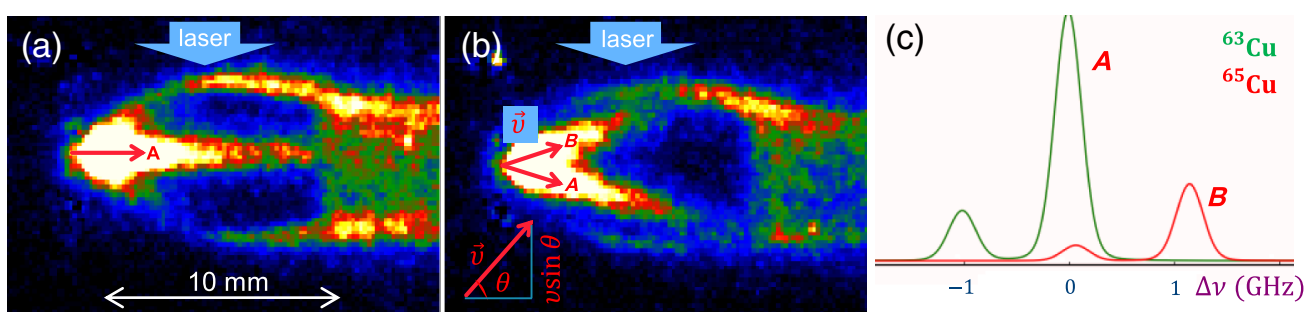

FIG. 9. Visualization of flow streamlines in the expanding flow of the supersonic free jet. $P_{0}=305 \mathrm{mbar}, P_{\mathrm{bg}}=0.9 \mathrm{mbar}$. Red arrows and the light blue arrow indicate flow streamlines and the direction of the laser beam, respectively. The start of the red arrows indicates the position of the nozzle exit. $\Delta \nu$ is the frequency detuning with respect to the frequency $\nu_{0}$. The color code indicates the intensity of the fluorescence signal in a similar way as in Fig. 7. (a) $\Delta \nu=0 \mathrm{GHz}$. (b) $\Delta \nu=+0.5 \mathrm{GHz}$. (c) Simulated spectra of the transitions from the hyperfine level $F=1$ of the ground state $4 s^{2} S_{1 / 2}$ to the hyperfine levels $F=1^{\prime}$ and $F=2^{\prime}$ of the excited state $4 p^{2} P_{1 / 2}$.

could not be neglected at small values of the $x$ coordinates. At $x=0.8 \mathrm{~mm}$, the Mach number $M=2.4$ [Eq. (11)] and the local pressure $P=25$ mbar [Eq. (14)]. The estimation of the upper limit of the collision broadening, assuming a collision broadening coefficient $\gamma_{\text {coll }}^{0}=10 \mathrm{MHz} / \mathrm{mbar}$ and no applied correction for the low temperatures in the jet, gave $250 \mathrm{MHz}$. The beam divergence contribution to the total linewidth was estimated to be $16 \mathrm{MHz}$, assuming
$T_{0}=450 \mathrm{~K}$ (see the next paragraph) with a corresponding velocity $v=555 \mathrm{~m} / \mathrm{s}$ at $M=2.4$ [Eq. (15)] and a length of the sampling pixel region of $0.22 \mathrm{~mm}$ in the direction perpendicular to the $x$ axis. For the next data point, at $x=1.2 \mathrm{~mm}$, the estimation of the upper limit of the collision and beam divergence broadenings gave 90 and $8 \mathrm{MHz}$, respectively. These collision broadenings should not be neglected and, thus, data points at $x=0.8$ and (a)

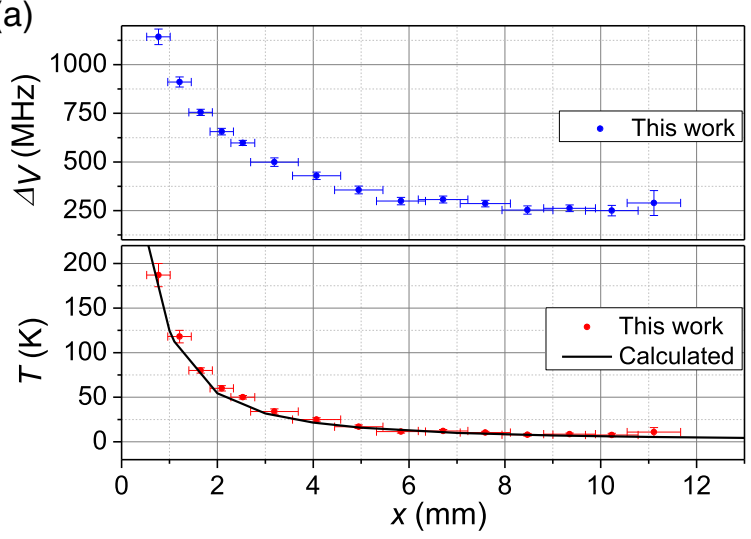

(c)
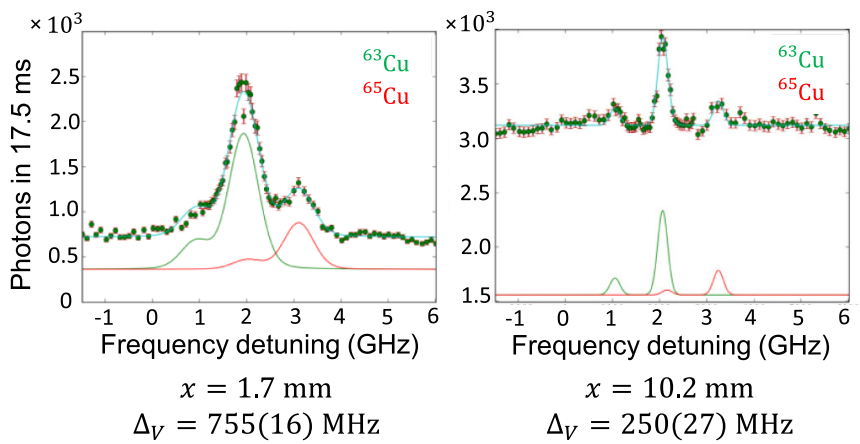

(b)

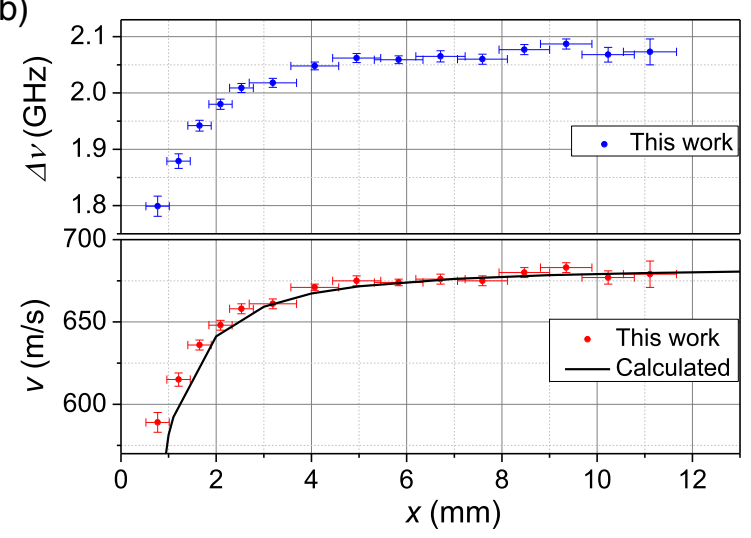

(d)

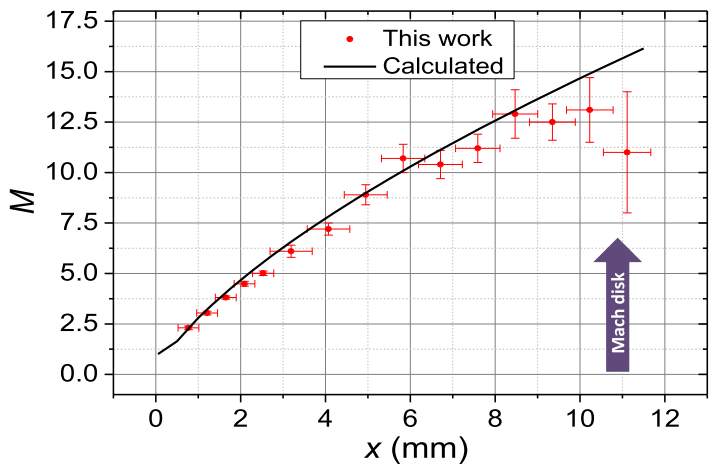

FIG. 10. Variations of the local characteristics along the central jet line in the expanding flow of the supersonic free jet. $P_{0}=355$ mbar, $P_{\mathrm{bg}}=1.3$ mbar. (a) Linewidth $\Delta_{V}$ and temperature $T$. (b) Doppler shift $\Delta \nu$ and velocity $v$. (c) The hyperfine spectra of the transitions from the hyperfine level $F=1$ of the ground state $4 s^{2} S_{1 / 2}$ to the hyperfine levels $F=1^{\prime}$ and $F=2^{\prime}$ of the excited state $4 p^{2} P_{1 / 2}$ in ${ }^{63,65} \mathrm{Cu}$ at two $x$ coordinates from the nozzle exit. (d) Mach number $M$. Calculated black curves show the outcome after using Eq. (10) [and Eqs. (11) and (12) for $x / d^{*}<1$ ] as well as Eqs. (13) and (15) $\left(T_{0}=450 \mathrm{~K}\right)$. 
$1.2 \mathrm{~mm}$ in Fig. 10 might give rise to erroneous values of the temperature and the velocity. For the data point at $x=1.7 \mathrm{~mm}$, the estimation of the upper limit of the collision and beam divergence broadenings gave 26 and $4 \mathrm{MHz}$, respectively. These collision and beam divergence broadenings could be neglected and, thus, the data points at $x \geq 1.7 \mathrm{~mm}$ in Fig. 10 gave reliable values for both temperature and velocity.

The smallest spectral linewidth reached in the expanding flow of the supersonic free jet is $\Delta_{V}=250(27) \mathrm{MHz}$. A transition to the free molecular regime was not reached in this measurement due to the Mach disk positioned at $x_{M} \approx 11 \mathrm{~mm}$ [Eq. (18)], while the terminal Mach number $M_{t}=35$, corresponding to a pressure $P_{0}=355 \mathrm{mbar}$ and $d^{*}=1 \mathrm{~mm}$, would be reached at a distance $x_{t}=35 \mathrm{~mm}$. In this case, only the continuous flow regime was present in the expanding flow of the supersonic free jet and no freezing of the axial Mach number could be observed. The measured Doppler shift reached $\Delta \nu=2.077(9) \mathrm{GHz}$ at $x=8.5 \mathrm{~mm}$, which corresponds to a velocity $v=$ $680(3) \mathrm{m} / \mathrm{s}$. The measured Mach number was 12.9(1.2) at $x=8.5 \mathrm{~mm}$. At this Mach number the flow velocity nearly reached its maximum value $(99 \%$ of the velocity at $M \gg 10)$ and could be used to extract the temperature $T_{0}$ by introducing Eq. (13) to Eq. (15). This resulted in a stagnation temperature $T_{0}=450(4) \mathrm{K}$, which is significantly higher than room temperature due to the heating of the argon gas by the filament $F 2$ installed $24 \mathrm{~mm}$ upstream from the nozzle throat. A numerical calculation using the CFD module was performed and showed that for filament $F 2$, in contrast to filament $F 1$, the gas heated by the filament does not have time to cool down and its temperature close to the nozzle position is $\sim 500 \mathrm{~K}$, in line with the experimental observations.

The concentration of copper atoms in the argon flow was very low and thus its influence on the argon flow parameters, such as local velocities and temperatures, was considered to be negligible. The measured dependence of the Mach number on the distance from the nozzle exit for the copper atoms seeded in argon agreed well with the results obtained for the supersonic free jets [59].

The good agreement between the earlier results from the literature and those obtained in this work by atomic PLIF spectroscopy supports the validity of the latter to study the thermodynamic and flow dynamic properties of the jets formed by de Laval nozzles.

\section{CHARACTERIZATION OF JETS FORMED BY DE LAVAL NOZZLES}

In order to gain an in-depth understanding of the flow properties of the jets formed by de Laval nozzles and of the parameters that are essential for the high spectral resolution and efficiency of the in-gas-jet method, a series of systematic measurements on the jet formation was carried out.

\section{A. Relative density profiles. Formation of long and uniform jets}

The optimization of the inner contour of de Laval nozzles is necessary for the formation of long jets with uniform flow parameters, such as velocity $v$, temperature $T$, and density $\rho$, and with a constant jet diameter [70]. This was demonstrated with numerical calculations of the jet formed by nozzle 1 (see Sec. II B).

Besides that, the formation of long, collimated, and uniform jets depends on the adjustment of the pressure $P_{\mathrm{bg}}$ inside the gas cell chamber. A mismatch of the pressure at the nozzle exit $P_{\text {exit }}$ with the pressure $P_{\text {bg }}$ results in the formation of overexpanded $\left(P_{\mathrm{bg}}>P_{\text {exit }}\right)$ or underexpanded $\left(P_{\mathrm{bg}}<P_{\text {exit }}\right)$ jets in which the jet pressure is adapted to the pressure $P_{\mathrm{bg}}$ through a series of shock cells and expansion waves [50]. In this case, the significant variations of the flow parameters can be observed, with higher densities and temperatures and lower velocities at the shock positions.

The visualization of the jet structure for the quasiuniform, over- and underexpanded jets was performed for nozzle 1 and nozzle 2 in transverse direction with the broadband laser (Fig. 11). In the case of underexpanded jets, variations of the jet diameter along the central jet line were observed. In the case of overexpanded jets, the formation of $M>1$ jets was not possible when the pressure $P_{\text {bg }}$ was too high. In this instance, shocks formed inside the nozzle's divergent part prohibited the formation of supersonic jets.

Variations of the normalized fluorescence signal, i.e., of the relative jet density, along the central jet line are shown in Fig. 12 for nozzle 1 and nozzle 2 for different values of the pressure $P_{\mathrm{bg}}$. The measurements were performed in longitudinal direction with the broadband laser. For nozzle 1 , fluctuations were minimized at the pressure ratio $P_{0} / P_{\mathrm{bg}}=232\left(P_{\mathrm{bg}}=1.4 \mathrm{mbar}\right)$ to a value of $\Xi=44 \%$ and had a period $\Lambda \approx 19 \mathrm{~mm}$ [see Fig. 12(a)]. The measured values of $\Xi$ and $\Lambda$ agreed well with that from the numerical calculations performed for nozzle 1 (see Table I). However, on top of the periodic structures, nonperiodic ones were observed in the fluorescence signal profiles and the fluctuations did not reduce further when scanning the value of $P_{\mathrm{bg}}$ with a fine step of $0.1 \mathrm{mbar}$. This indicates that other sources of flow nonuniformities were present apart from the pressure mismatch, such as an erroneous optimization of the inner contour that resulted in formation of shock waves which are impossible to eliminate [see color map of Mach number in Fig. 3(a)] or an imperfect manufacturing of the inner nozzle's contour. For nozzle 2, whose inner contour was optimized using the CFD module, the signal fluctuations were minimized at the pressure ratio $P_{0} / P_{\mathrm{bg}}=35\left(P_{\mathrm{bg}}=9.7 \mathrm{mbar}\right)$ to $\Xi=26 \%$ with a periodicity $\Lambda=6 \mathrm{~mm}$ [Fig. 12(b)]. The experimental fluctuations were larger than those obtained with the numerical calculations in Sec. II B. The optimization of the 
(a)
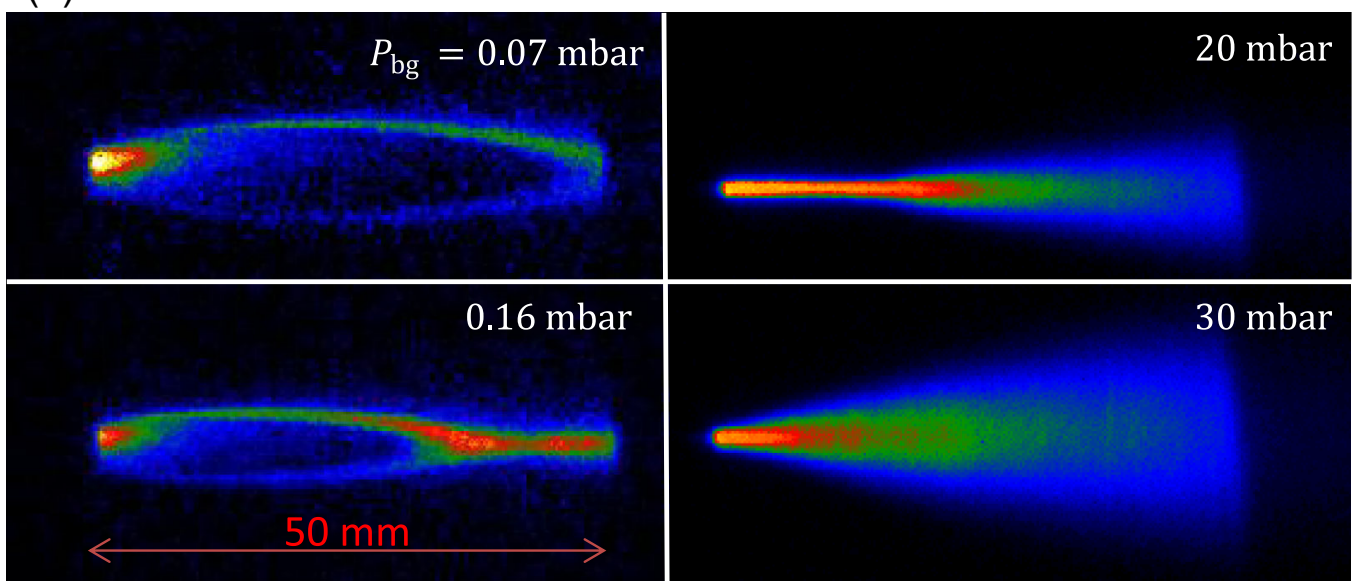

(b)
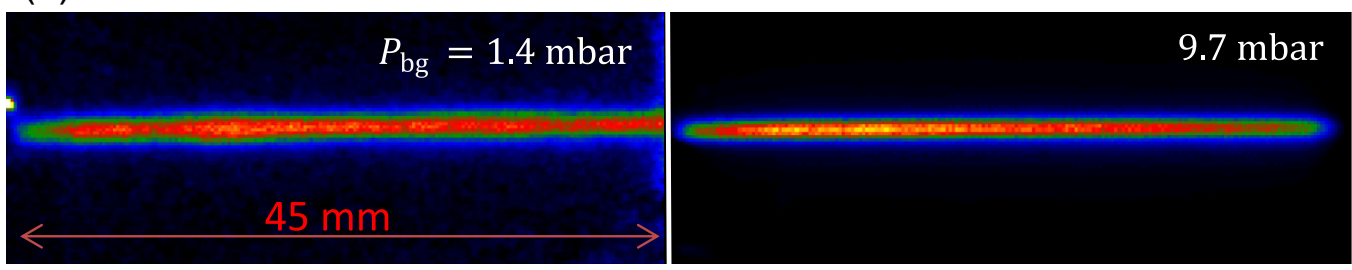

FIG. 11. Visualization of quasiuniform, over- and underexpanded jets. The color code indicates the intensity of the fluorescence signal in a similar way as in Fig. 7. (a) Under- and overexpanded jets formed by nozzle 1 are shown in the left-hand and right-hand panels, respectively, for $P_{0} \sim 300$ mbar. (b) Quasiuniform jets formed by nozzle 1 for a pressure $P_{0} \sim 325$ mbar (left-hand panel) and nozzle 2 for $P_{0} \sim 340$ mbar (right-hand panel) for adjusted pressures $P_{\mathrm{bg}}$ inside the gas cell chamber.

inner contour of nozzle 2 was performed for the temperature $T_{0}=300 \mathrm{~K}$ at the nozzle inlet, while in the experiment $T_{0}$ was higher, owing to the heating of the argon buffer gas by filament $F 2$ (see Sec. III). Nozzle 2 was thus utilized at off-design operating conditions, which led to larger fluctuations $\Xi$. Additionally, it is interesting to note that an increase of the pressure $P_{\text {bg }}$ by a factor of $\sim 2.3$

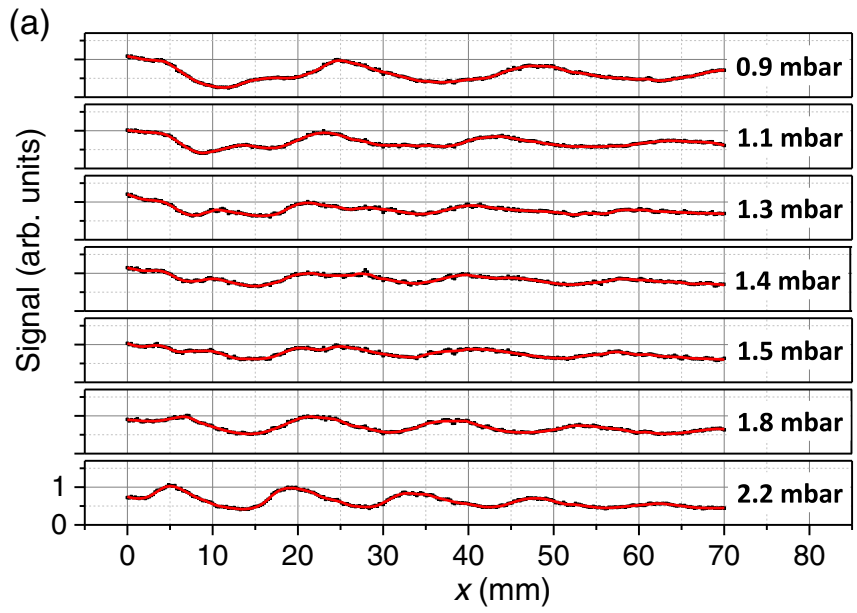

caused more changes in the fluorescence signal profiles at longer distances from the nozzle exit than the decrease of $P_{\text {bg }}$ by the same factor.

The visualization of the central jet line proved that the formation of long $(>80 \mathrm{~mm})$ jets with quasiuniform fluorescence signal profiles and constant jet diameters is possible with the nozzles used in our studies.

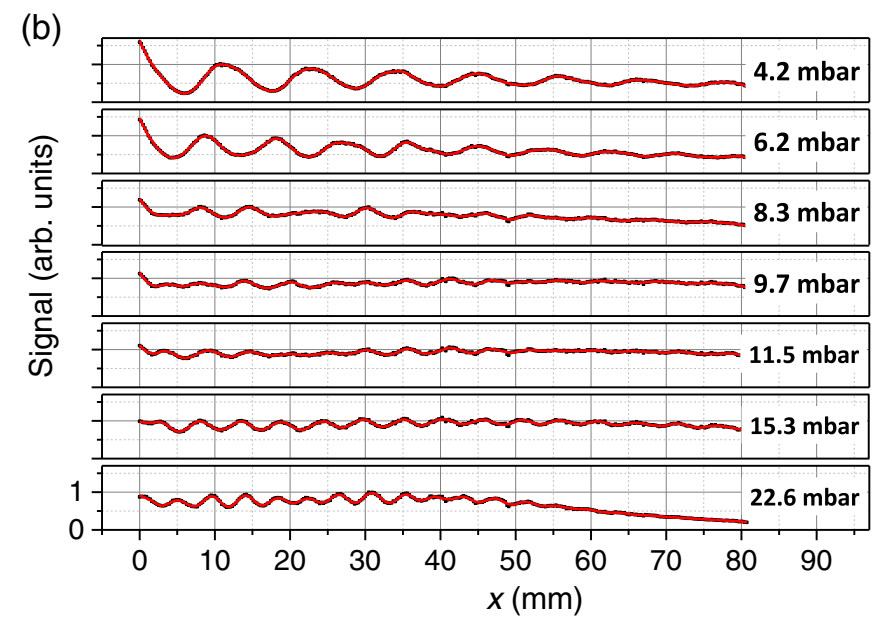

FIG. 12. Variations of the normalized fluorescence signal along the central jet line for different values of the pressure $P_{\text {bg }}$, indicated in the graphs. Red curves show Savitzky-Golay signal processing [71] of PLIF measurements data (black symbols). (a) Nozzle 1. $P_{0} \sim 325$ mbar. (b) Nozzle 2. $P_{0} \sim 340$ mbar. 
B. Velocity, temperature, and Mach number profiles. Narrow band spectroscopy with the jets formed by de Laval nozzles

Velocity, temperature, and Mach number profiles of the jets formed by nozzle 1 were extracted from PLIF spectroscopy studies performed in longitudinal direction with the narrow band laser for the cases of an underexpanded jet (Fig. 13) and a quasiuniform jet (Fig. 14). For the latter, the pressure ratio $P_{0} / P_{\mathrm{bg}}$ was adjusted to be close to the optimal value [see Fig. 12(a)]. The narrow band scans were performed with a laser energy fluence $\Phi \sim 10 \mathrm{pJ} / \mathrm{mm}^{2}$.

(a)

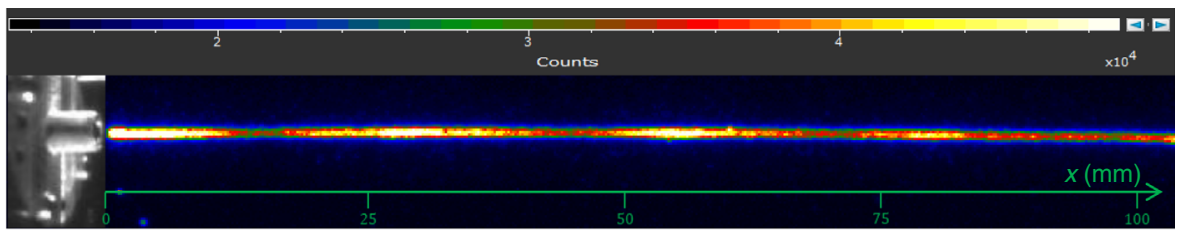

(b)
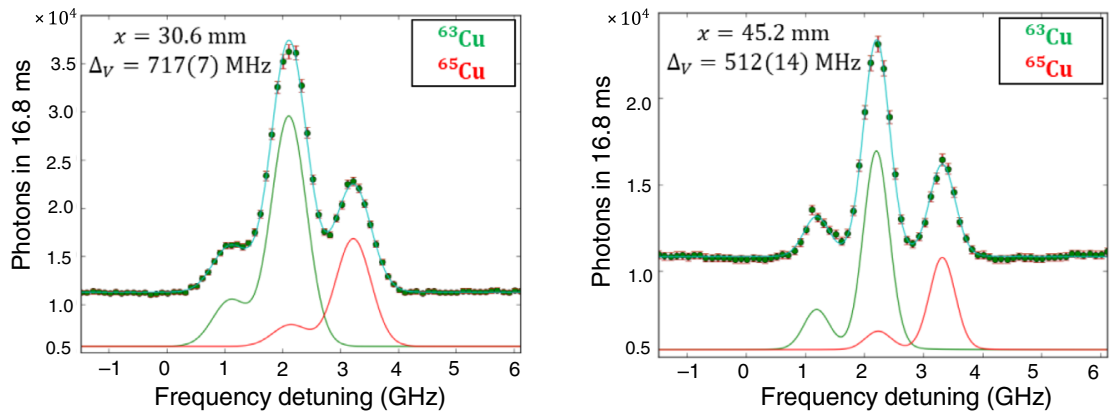

(c)

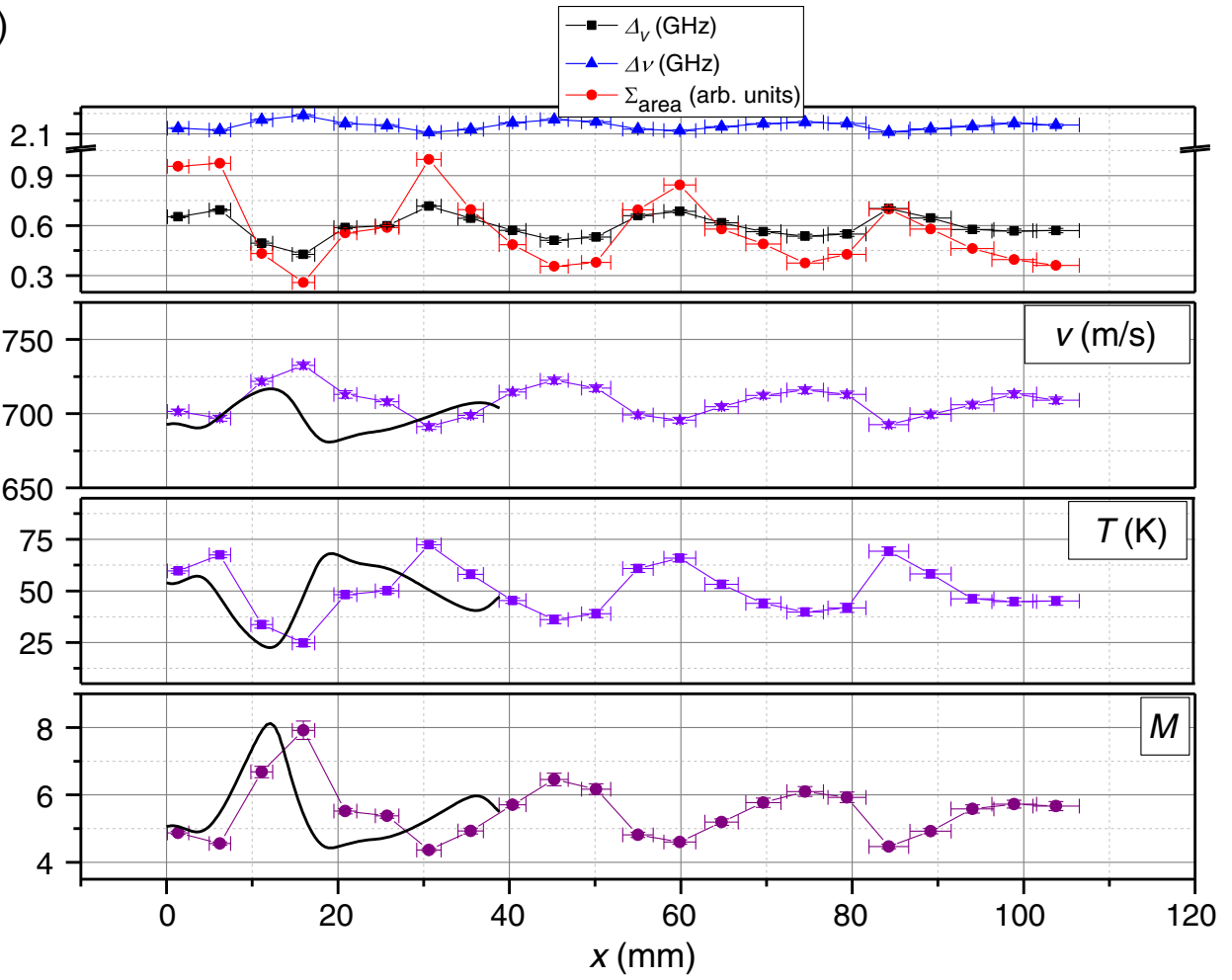

FIG. 13. Characterization of an underexpanded jet formed by nozzle 1. $P_{0}=389 \mathrm{mbar}, P_{\mathrm{bg}}=0.79$ mbar. (a) The central jet line of the underexpanded jet with the color code indicating the intensity of the fluorescence signal. (b) The measured hyperfine spectra of the transitions from the hyperfine level $F=1$ of the ground state $4 s^{2} S_{1 / 2}$ to the hyperfine levels $F=1^{\prime}$ and $F=2^{\prime}$ of the excited state $4 p^{2} P_{1 / 2}$ in ${ }^{63,65} \mathrm{Cu}$ at two $x$ coordinates from the nozzle exit. (c) The variations of the local jet characteristics, such as spectroscopic parameters (linewidth $\Delta_{V}$ and Doppler shift $\Delta \nu$ ) integral of the area below the fit $\Sigma_{\text {area }}$, velocity $v$, temperature $T$, and Mach number $M$, along the central jet line are shown. The numerical calculations using the CFD module were performed for $T_{0}=520 \mathrm{~K}, P_{0}=389$ mbar, and $P_{\mathrm{bg}}=0.79$ mbar (black curves). 
(a)

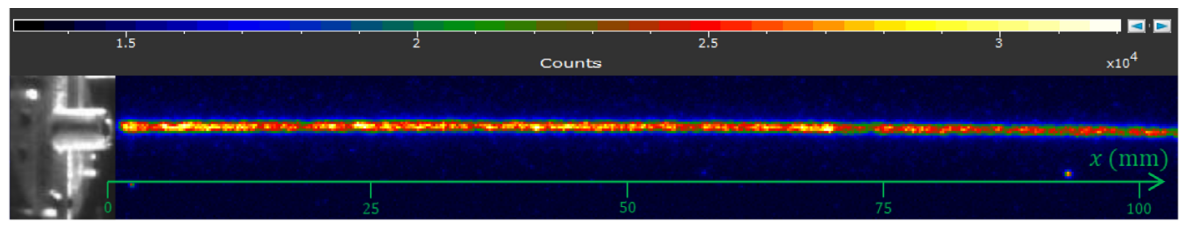

(b)
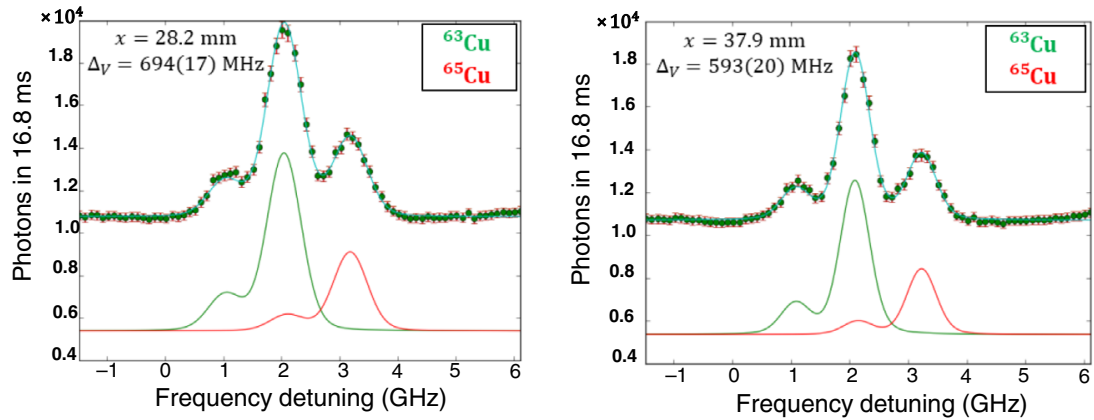

(c)
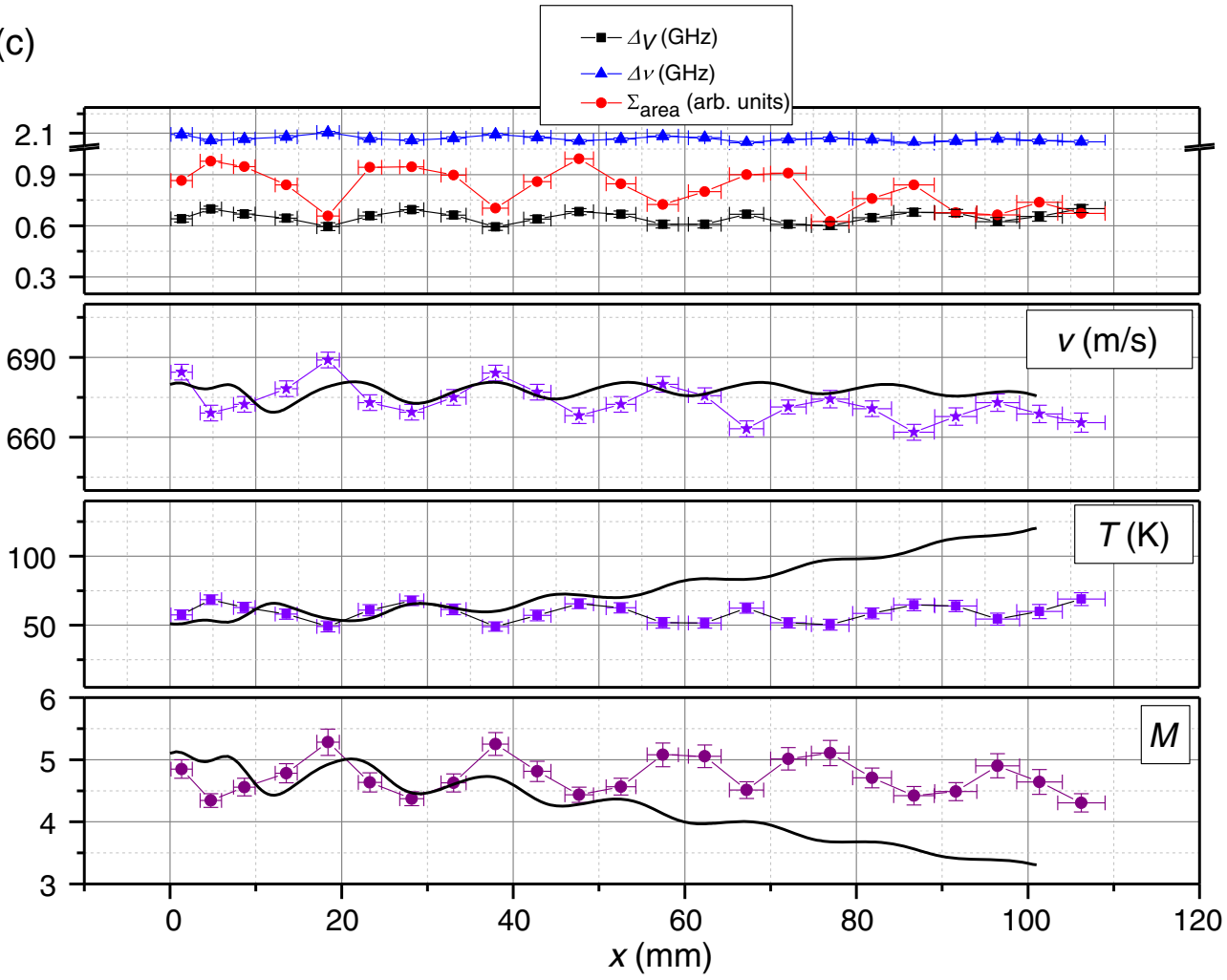

FIG. 14. Characterization of a jet formed by nozzle 1 at near-optimal pressure $P_{\mathrm{bg}} . P_{0}=379 \mathrm{mbar}, P_{\mathrm{bg}}=1.46 \mathrm{mbar}$. (a) The central jet line of the quasiuniform jet with the color code indicating the intensity of the fluorescence signal. (b) The measured hyperfine spectra of the transitions from the hyperfine level $F=1$ of the ground state $4 s^{2} S_{1 / 2}$ to the hyperfine levels $F=1^{\prime}$ and $F=2^{\prime}$ of the excited state $4 p^{2} P_{1 / 2}$ in ${ }^{63,65} \mathrm{Cu}$ at two $x$ coordinates from the nozzle exit. (c) The variation of the local jet characteristics, such as spectroscopic parameters (linewidth $\Delta_{V}$ and Doppler shift $\Delta \nu$ ) integral of the area below the fit $\Sigma_{\text {area }}$, velocity $v$, temperature $T$, and Mach number $M$ along the central jet line, are shown. The numerical calculations using the CFD module were performed for $T_{0}=500 \mathrm{~K}$, $P_{0}=379$ mbar, and $P_{\mathrm{bg}}=1.46$ mbar (black curves).

Strong variations of the local jet parameters along the central jet line were observed for the underexpanded jet, as shown in Fig. 13. For the linewidth $\Delta_{V}$, these variations ranged from 450 to $750 \mathrm{MHz}$, for the extracted velocity $v$ from 680 to $730 \mathrm{~m} / \mathrm{s}$, for the temperature $T$ from 25 to $75 \mathrm{~K}$ and for the Mach number $M$ from 4.5 to 8 . An integral of the area under the Voigt fit $\Sigma_{\text {area }}$, which reflects the density variations, was extracted. The jet regions with smaller values of the integral $\Sigma_{\text {area }}$, and thus with smaller local densities, had a smaller linewidth $\Delta_{V}$ and a larger Doppler shift $\Delta \nu$ (or, equivalently, lower temperatures and higher velocities). Additionally, the spectral linewidth 
(Voigt FWHM) and Doppler shift were extracted from fitting the variations of the light intensity collected over the whole length of the central jet line as a function of the laser frequency, resulting in averaged values $\overline{\Delta_{V}}=$ 618(12) $\mathrm{MHz}$ and $\overline{\Delta \nu}=2.158(7) \mathrm{GHz}$, respectively. The variations of the local jet parameters along the central jet line were reduced when the quasiuniform jet was formed (Fig. 14). $\Delta_{V}$ ranged from 600 to $700 \mathrm{MHz}, v$ from 660 to $690 \mathrm{~m} / \mathrm{s}, T$ from 50 to $70 \mathrm{~K}$, and $M$ from 4.5 to 5.5. The spectral linewidth $\overline{\Delta_{V}}$ was $656(20) \mathrm{MHz}$ and the Doppler shift $\overline{\Delta \nu}$ was $2.055(9) \mathrm{GHz}$. The errors of $\overline{\Delta_{V}}$ and $\overline{\Delta \nu}$ are larger in the measurement with the quasiuniform jet, as it was performed with a slightly lower filament current, giving rise to a smaller fluorescence signal and thus to lower statistics.

The measured velocities were higher than $550 \mathrm{~m} / \mathrm{s}$ because of the close position of the filament utilized in these measurements to the nozzle throat, which resulted in the heating of the argon buffer gas close to the nozzle position (see Sec. III) and thus in a higher jet velocity. For the in-gas-jet method, a higher jet velocity would imply the use of a laser with a higher repetition rate $f>10 \mathrm{kHz}$ or the formation of a longer laser sheet $l>55 \mathrm{~mm}$ to match the duty cycle and thus keep the high efficiency of the method. However, the implementation of the method in online conditions is foreseen at lower temperatures $T_{0}$ close to $300 \mathrm{~K}$, since the filament will be used only for very short periods of time, if any, or the production of stable atoms will be performed by laser ablation rather than by a glowing filament.

The observed difference of $\overline{\Delta \nu}$ between the quasiuniform and underexpanded jets was further examined with numerical calculations using the CFD module showing that the best agreement between the calculations and the experiment was achieved for $T_{0}=520 \mathrm{~K}$ for the underexpanded jet and for $T_{0}=500 \mathrm{~K}$ for the quasiuniform jet at $x$ coordinates close to the nozzle exit (see black curves for velocity, temperature, and Mach number in Figs. 13 and 14). Moreover, the temperature of the outer wall of the stainless steel gas cell was measured with a PT100 sensor and found to be $75^{\circ} \mathrm{C}$ and $68^{\circ} \mathrm{C}$ at the end of the PLIF spectroscopy measurements for the underexpanded and quasiuniform jets, respectively. For the underexpanded jet (Fig. 13), the numerical calculations were performed for $x<40 \mathrm{~mm}$ due to problems with numerical solver convergence when calculating longer jets at nonoptimal pressure $P_{\mathrm{bg}}$. The observed shift between the experimental and the numerical calculations results is likely to be caused by a small deviation of the manufactured inner contour of nozzle 1 from that used in the CFD module, which caused a shift of shocks structure. For the quasiuniform jet (Fig. 14), a disagreement between the experimental and the numerical calculations results for the temperature and Mach number at $x>60 \mathrm{~mm}$ is expected to be caused by an erroneous evaluation of viscosity and heat conductivity at low temperatures and/or by the conditions at the outflow boundaries in the CFD module and thus requires more research to be conducted.

The spectral linewidth $\overline{\Delta_{V}}$ fairly agreed within error bars for the two considered cases of the pressure $P_{\mathrm{bg}}$. Nevertheless, the drawback for the in-gas-jet method to be implemented with underexpanded jets is the increased and varying diameter of the jets that imposes higher requirements on the laser energy in order to keep a high ionization efficiency. For instance, an increase of the jet diameter by a factor of 2 would require 4 times higher laser energy.

At sufficiently large distances from the nozzle exit, the mixing of the argon gas jet with the ambient argon gas is expected to cause an increase of the jet temperature and a decrease of the jet density, both of which will approach the ambient conditions [31]. This effect, however, was not observed in our experiments for copper seeded to the flow up to an axial distance of $100 \mathrm{~mm}$ from the nozzle.

More systematic studies with nozzle 3 and nozzle 4 were initiated on the influence of other factors, such as the temperature inside the gas cell $T_{0}$ and the finishing of the nozzle inner contour, on the flow parameters. The measurements were performed with the value of the pressure $P_{\mathrm{bg}}$ optimized in each case to form quasiuniform jets. A summary of the results obtained with PLIF spectroscopy studies using these nozzles is presented in Table II. The spectral linewidth $\overline{\Delta_{V}}$ obtained with the filament $F 1$ was enhanced owing to the lower temperature $T_{0}$. The extracted jet velocity is $\bar{v}=532(32) \mathrm{m} / \mathrm{s}$, as expected for

TABLE II. Characterization of the flow properties of jets formed by de Laval nozzles for few cases of the designed nozzle geometry, surface roughness of the inner contour $R_{a}$, and the temperature inside the gas cell $T_{0}$ (or, equally, which of the filaments $F 1$ or $F 2$ was used to feed copper into the argon flow). The values of the Doppler shift $\overline{\Delta \nu}$, spectral linewidth $\overline{\Delta_{V}}$, velocity $\bar{v}$, temperature $\bar{T}$, and Mach number $\bar{M}$ are presented. The surface roughness of the inner contour of nozzle 1 was not measured within these studies.

\begin{tabular}{lcclllll}
\hline \hline Nozzles & $R_{a}(\mu \mathrm{m})$ & Filament & $\overline{\Delta \nu}(\mathrm{GHz})$ & $\overline{\Delta_{V}}(\mathrm{MHz})$ & $\bar{v}(\mathrm{~m} / \mathrm{s})$ & $\bar{T}(\mathrm{~K})$ & $\bar{M}$ \\
\hline Nozzle 1 & $\ldots$ & $F 2$ & $2.055(9)$ & $656(20)$ & $673(3)$ & $60(4)$ & $4.65(15)$ \\
Nozzle 3 & 0.8 & $F 2$ & $2.088(17)$ & $611(25)$ & $684(6)$ & $52(4)$ & $5.1(2)$ \\
Nozzle 4 & 0.4 & $F 2$ & $2.051(6)$ & $464(14)$ & $672(2)$ & $29(2)$ & $6.7(2)$ \\
Nozzle 4 & 0.4 & $F 1$ & $1.60(10)$ & $339(148)$ & $532(35)$ & $15(14)$ & $7(4)$ \\
\hline \hline
\end{tabular}


$T_{0} \sim 300 \mathrm{~K}$. PLIF spectroscopy showed formation of jets of the same (within the error bars) Mach number $\bar{M}$ for the two cases of the temperature $T_{0}$. However, the error bar of the measurement with filament $F 1$ is large due to the low fluorescence signal. This error can be reduced when performing more sensitive spectroscopy studies using resonance ionization spectroscopy. Moreover, experimental results showed a dependency of the Mach number of the formed jets on the finishing of the inner contour of the nozzle for the two values of the tested surface roughness $R_{a}$ of 0.8 and $0.4 \mu \mathrm{m}$. This dependency can be explained by the strong influence of the surface roughness at the very beginning of the divergent part of the nozzle, where the boundary layer is very thin, on the jet formation.

Finally, the temperature of the inner contour of nozzle 3 manufactured out of stainless steel was measured with the PT100 sensor installed in such a way that the supersonic flow was not disturbed. A drop of less than $1{ }^{\circ} \mathrm{C}$ was observed during three hours of temperature monitoring with a continuous argon flow corresponding to the working conditions of $P_{0}=300$ mbar.

\section{CONCLUSIONS AND OUTLOOK}

An exhaustive characterization of the supersonic gas jets formed by a number of de Laval nozzles was performed at the IGLIS laboratory using PLIF spectroscopy on copper atoms. Velocity, temperature, Mach number, and relative density profiles of argon jets were extracted with a high precision. Error bars were found to be about $\pm 3 \mathrm{~m} / \mathrm{s}$ for the velocity and $\pm 4 \mathrm{~K}$ for the temperature in the jet in the cases with optimal fluorescence signal (see Figs. 13 and 14). The optimization and manufacturing of the nozzle's inner contour and the pressure matching between the nozzle exit and the background medium are principal for the formation of long $(>80 \mathrm{~mm})$ quasiuniform jets and thus for a high spectral resolution and efficiency of the in-gas-jet method. A further minimization of the deviation tolerance of the inner contour and its measurements with the Werth fiber probe (see Fig. 4) are the objectives for the next nozzles to be manufactured and tested. On the other hand, the use of higher repetition rate lasers $(>10 \mathrm{kHz})$ can mitigate the requirements for the quasiuniformity of the jets, since the optimal temporal overlap between the laser pulses and the high-velocity jet can be fulfilled on a carefully selected shorter region of the jet, the flow properties of which will match the requirements for the high-resolution spectroscopy studies.

The smallest spectral linewidth reached in these studies was $\overline{\Delta_{V}}=464(14) \mathrm{MHz}$ (see Table II), with optimal fluorescence signal and optimized value of a pressure $P_{\mathrm{bg}}$ for the formation of a quasiuniform jet. The corresponding temperature-associated broadening was $\Delta \nu_{\text {Doppler }} \sim$ $450 \mathrm{MHz}$ for the ${ }^{63,65} \mathrm{Cu}$ isotopes. The test was performed with filament $F 2$ for which the numerical calculations showed a gas temperature close to the nozzle position was $T_{0}>300 \mathrm{~K}$. An expected linewidth reduction will be feasible following the steps discussed below and summarized in Table III.

(1) Implementing the in-gas-jet method in on-line conditions where the preselected RIBs from a particle accelerator are thermalized inside the gas cell via collisions with the buffer gas to temperatures of $300 \mathrm{~K}$.

(2) Applying the in-gas-jet method on the actinides will result in an additional reduction of the linewidths as compared to that obtained for the copper isotopes as the broadening $\Delta \nu_{\text {Doppler }}$ is inversely proportional to the square root of the atomic mass of the isotopes under the study.

(3) Utilizing de Laval nozzles with higher Mach numbers will result in lower jet temperatures and densities and thus in a reduction of the associated broadenings mechanisms. The maximum accessible Mach number $M_{\max }$ depends on the employed vacuum system capabilities and on the nozzle throat diameter $d^{*}$ (mass flow). For example, $M_{\max } \sim 8.5$ for $d^{*}=1 \mathrm{~mm}$ with the screw pump GXS 450/4 200 (890 1/s) from Edwards Vacuum [46]. Moreover, implementation of de Laval nozzles with high Mach number reduces the required background pressure $\left(P_{\mathrm{bg}} \sim 0.1 \mathrm{mbar}\right.$ for $P_{0}=300 \mathrm{mbar}$ and $M=8.5$ ). This allows us to operate the RFQ structures (see, e.g., Fig. 2) below the Paschen curve to avoid sparking.

As demonstrated in this paper, the PLIF method allows us to test new designs of de Laval nozzles towards a Mach number of 8.5, which is at the operational limit of the $S^{3}$-LEB setup [25]. For the ${ }^{253}$ No experiment, this will result in a spectral linewidth of about $140 \mathrm{MHz}$ (see Table III) to be compared to the $4 \mathrm{GHz}$ obtained in a

TABLE III. Projected spectral linewidth of the in-gas-jet laser ionization and spectroscopy technique as, e.g., implemented in the $S^{3}$-LEB setup [25].

\begin{tabular}{lccccc}
\hline \hline Isotope & Electronic transition & $T_{0}(\mathrm{~K})$ & Mach & $T(\mathrm{~K})$ & $\Delta \nu_{\text {Doppler }}(\mathrm{MHz})$ \\
\hline${ }^{63} \mathrm{Cu}$ & $4 s{ }^{2} S_{1 / 2} \rightarrow 4 p^{2} P_{1 / 2} 327.40 \mathrm{~nm}$ & $\sim 465$ & 6.7 & 29 & 450 \\
${ }^{63} \mathrm{Cu}$ & $4 s{ }^{2} S_{1 / 2} \rightarrow 4 p{ }^{2} P_{1 / 2} 327.40 \mathrm{~nm}$ & 300 & 6.7 & 19 & 360 \\
${ }^{253} \mathrm{No}$ & $7 s^{2}{ }^{1} S_{0} \rightarrow 7 s 7 p{ }^{1} P_{1} 333.76 \mathrm{~nm}$ & 300 & 6.7 & 19 & 175 \\
${ }^{253} \mathrm{No}$ & $7 s^{2}{ }^{1} S_{0} \rightarrow 7 s 7 p{ }^{1} P_{1} 333.76 \mathrm{~nm}$ & 300 & 8.5 & 12 & 140 \\
\hline \hline
\end{tabular}


recent in-gas cell study [72]. With the enhanced spectral resolution of the in-gas-jet method, the uncertainties on the nuclear ground-state properties will no longer be dominated by experimental errors, but by the uncertainties from atomic theory.

In conclusion, the high spectral resolution, combined with the high efficiency of the in-gas-jet method, enables study of the nuclear ground- and isomeric-state properties of the actinides produced at a small rate of 1 atom every $10 \mathrm{~s}$, pushing further the limits of the laser ionization spectroscopy studies of the nuclear structure.

\section{ACKNOWLEDGMENTS}

This work has been funded by FWO-Vlaanderen (Belgium), by GOA/2015/010 (BOF KU Leuven), by the Interuniversity Attraction Poles Programme initiated by the Belgian Science Policy Office (BriX network P7/12), and by a grant from the European Research Council (ERC2011-AdG-291561-HELIOS). This project has received funding from the European Union's Horizon 2020 research and innovation programme under Grant Agreement No. 654002. L. P. G. acknowledges FWO Vlaanderen (Belgium) as an FWO Pegasus Marie Curie Fellow. P. P. acknowledges the People Programme (Marie Curie Actions) of the European Union's Seventh Framework Programme (FP7/2007-2013) under REA Grant Agreement No. 626518.

[1] P. Campbell, I. D. Moore, and M. R. Pearson, Laser Spectroscopy for Nuclear Structure Physics, Prog. Part. Nucl. Phys. 86, 127 (2016).

[2] T. Bürvenich, K. Rutz, M. Bender, P.-G. Reinhard, J. A. Maruhn, and W. Greiner, Superheavy Nuclei in Deformed Mean-Field Calculations, Eur. Phys. J. A 3, 139 (1998).

[3] M. Bender, K. Rutz, P.-G. Reinhard, J. A. Maruhn, and W. Greiner, Potential Energy Surfaces of Superheavy Nuclei, Phys. Rev. C 58, 2126 (1998).

[4] Yu. Ts. Ogannessian, Nuclear Physics: Sizing Up the Heavyweights, Nature (London) 413, 122 (2001).

[5] Special issue on Superheavy Elements, edited by C. E. Düllmann, R.-D. Herzberg, W. Nazarewicz, and Y. Oganessian [M. Asai, F. P. Heßberger, and A. Lopez-Martens, Nuclear Structure of Elements with $100 \leq Z \leq 109$ from Alpha Spectroscopy, Nucl. Phys. A944, 308 (2015)].

[6] P. T. Greenlees et al., In-Beam and Decay Spectroscopy of Transfermium Elements, Eur. Phys. J. A 25, 599 (2005).

[7] Y. T. Oganessian et al., Synthesis of the Isotopes of Elements 118 and 116 in the ${ }^{249} \mathrm{Cf}$ and ${ }^{245} \mathrm{Cm}+{ }^{48} \mathrm{Ca}$ Fusion Reactions, Phys. Rev. C 74, 044602 (2006).

[8] Y. T. Oganessian et al., Production and Decay of the Heaviest Nuclei ${ }^{293,294} 117$ and ${ }^{294} 118$, Phys. Rev. Lett. 109, 162501 (2012).

[9] T. K. Sato et al., Measurement of the First Ionization Potential of Lawrencium, Element 103, Nature (London) 520, 209 (2015).
[10] M. Laatiaoui et al., Atom-at-a-Time Laser Resonance Ionization Spectroscopy of Nobelium, Nature (London) 538, 495 (2016).

[11] A. Săndulescu, R. K. Gupta, W. Scheid, and W. Greiner, Synthesis of New Elements within the Fragmentation Theory: Application to $Z=104$ and 106 Elements, Phys. Lett. B 60, 225 (1976).

[12] W. Greiner, Novel Clusters in Nuclear Physics: Superheavy, Superstrange, Antimatter, Eur. Phys. J. D 66, 200 (2012).

[13] K. Marinova, W. Geithner, M. Kowalska, K. Blaum, S. Kappertz, M. Keim, S. Kloos, G. Kotrotsios, P. Lievens, R. Neugart, H. Simon, and S. Wilbert, Charge Radii of Neon Isotopes Across the sd Neutron Shell, Phys. Rev. C 84, 034313 (2011).

[14] R. P. de Groote et al., Dipole and Quadrupole Moments of ${ }^{73-78} \mathrm{Cu}$ as a Test of the Robustness of the $Z=28$ Shell Closure Near ${ }^{78} \mathrm{Ni}$, Phys. Rev. C 96, 041302 (2017).

[15] V. N. Fedosseev, D. V. Fedorov, R. Horn, G. Huber, U. Köster, J. Lassen, V. I. Mishin, M. D. Seliverstov, L. Weissman, and K. Wendt, Atomic Spectroscopy Studies of ShortLived Isotopes and Nuclear Isomer Separation with the ISOLDE RILIS, Nucl. Instrum. Methods Phys. Res., Sect. B 204, 353 (2003).

[16] P. Van Duppen, Laser Ion Sources for On-Line Isotope Separators, Nucl. Instrum. Methods Phys. Res., Sect. B 126, 66 (1997).

[17] Yu. Kudryavtsev et al., Beams of Short Lived Nuclei Produced by Selective Laser Ionization in a Gas Cell, Nucl. Instrum. Methods Phys. Res. Progr. Part. 114, 350 (1996).

[18] M. Facina, B. Bruyneel, S. Dean, J. Gentens, M. Huyse, Yu. Kudryavtsev, P. Van den Bergh, and P. Van Duppen, A Gas Cell for Thermalizing, Storing and Transporting Radioactive Ions and Atoms. Part II: On-Line Studies with a Laser Ion Source, Nucl. Instrum. Methods Phys. Res., Sect. B 226, 401 (2004).

[19] Yu. Kudryavtsev, T. E. Cocolios, J. Gentens, M. Huyse, O. Ivanov, D. Pauwels, T. Sonoda, P. Van den Bergh, and P. Van Duppen, Dual Chamber Laser Ion Source at LISOL, Nucl. Instrum. Methods Phys. Res., Sect. B 267, 2908 (2009).

[20] T. E. Cocolios et al., Magnetic Dipole Moment of ${ }^{57,59} \mathrm{Cu}$ Measured by In-Gas-Cell Laser Spectroscopy, Phys. Rev. Lett. 103, 102501 (2009).

[21] R. Ferrer et al., In-Gas-Cell Laser Ionization Spectroscopy in the Vicinity of ${ }^{100} \mathrm{Sn}$ : Magnetic Moments and Mean-Square Charge Radii of N = 50-54 Ag, Phys. Lett. B 728, 191 (2014).

[22] C. Granados et al., In-Gas Laser Ionization and Spectroscopy of Actinium Isotopes Near the $N=126$ Closed Shell, Phys. Rev. C 96, 054331 (2017).

[23] Yu. Kudryavtsev, R. Ferrer, M. Huyse, P. Van den Bergh, and P. Van Duppen, The In-Gas-Jet Laser Ion Source: Resonance Ionization Spectroscopy of Radioactive Atoms in Supersonic Gas Jets, Nucl. Instrum. Methods Phys. Res., Sect. B 297, 7 (2013).

[24] R. Ferrer et al., Towards High-Resolution Laser Ionization Spectroscopy of the Heaviest Elements in Supersonic Gas Jet Expansion, Nat. Commun. 8, 14520 (2017).

[25] R. Ferrer et al., In Gas Laser Ionization and Spectroscopy Experiments at the Superconducting Separator Spectrometer (S3): Conceptual Studies and Preliminary Design, Nucl. Instrum. Methods Phys. Res., Sect. B 317, 570 (2013). 
[26] P. Papadakis, I. Moore, I. Pohjalainen, J. Sarén, and J. Uusitalo, Development of a Low-Energy Radioactive Ion Beam Facility for the MARA Separator, Hyperfine Interact. 237, 152 (2016).

[27] V. I. Zagrebaev, S. G. Zemlyanoy, E. M. Kozulin, Yu. Kudryavtsev, V. Fedosseev, R. Bark, Z. Janas, and H. A. Othman, Gas-Cell-Based Setup for the Production and Study of Neutron Rich Heavy Nuclei, Hyperfine Interact. 227, 181 (2014).

[28] Y. Hirayama et al., Doughnut-Shaped Gas Cell for KEK Isotope Separation System, Nucl. Instrum. Methods Phys. Res., Sect. B 412, 11 (2017).

[29] T. Sonoda, H. Iimura, M. Reponen, M. Wada, I. Katayama, V. Sonnenschein, T. Takamatsu, H. Tomita, and T. M. Kojima, The Laser and Optical System for the RIBF-PALIS Experiment, Nucl. Instrum. Methods Phys. Res., Sect. A 877, 118 (2018).

[30] E. Mach, Popular Scientific Lectures (The Open Court Publishing Company, Chicago, 1898).

[31] J. Panda and R. G. Seasholtz, Velocity and Temperature Measurement in Supersonic Free Jets Using Spectrally Resolved Rayleigh Scattering, in Proceedings of the 37th Aerospace Sciences Meeting and Exhibit, 1999, DOI: $10.2514 / 6.1999-296$.

[32] A. F. Mielke, K. A. Elam, and C.-J. Sung, Rayleigh Scattering Diagnostic for Measurement of Temperature, Velocity and Density Fluctuation Spectra, in Proceedings of the 44th AIAA Aerospace Sciences Meeting and Exhibit, 2006, Vol. 837, DOI: 10.2514/6.2006-837.

[33] R. J. Cattolica, R. J. Gallagher, J. B. Anderson, and L. Talbot, Aerodynamic Separation of Gases by Velocity Slip in Freejet Expansions, AIAA J. 17, 344 (1979).

[34] M. Belan, S. Massaglia, D. Tordella, M. Mirzaei, and S. de Ponte, The Hydrodynamics of Astrophysical Jets: Scaled Experiments and Numerical Simulations, Astron. Astrophys. 554, A99 (2013).

[35] C. W. Peterson, A Survey of the Utilitarian Aspects of Advanced Flowfield Diagnostic Techniques, AIAA J. 17, 1352 (1979).

[36] M. Rasi, R. Saintola, and K. Valli, Visualizing the Expanding Flow of Gas from Helium-Jet and Ion-Guide Nozzles, Nucl. Instrum. Methods Phys. Res., Sect. A 378, 251 (1996).

[37] M. Reponen, I. D. Moore, I. Pohjalainen, T. Kessler, P. Karvonen, J. Kurpeta, B. Marsh, S. Piszczek, V. Sonnenschein, and J. Äystö, Gas Jet Studies Towards an Optimization of the IGISOL LIST Method, Nucl. Instrum. Methods Phys. Res., Sect. A 635, 24 (2011).

[38] N. T. Clemens, Encyclopedia of Imaging Science and Technology (John Wiley and Sons, New York, 2002), pp. 390-419.

[39] S. O’Byrne, P. Danehy, and A. Houwing, Nonintrusive Temperature and Velocity Measurements in a Hypersonic Nozzle Flow, in Proceedings of the 22nd AIAA Aerodynamic Measurement Technology and Ground Testing Conference, Fluid Dynamics and Co-located Conferences, 2002, Vol. 2917, DOI: 10.2514/6.2002-2917.

[40] M. P. Lee, P. H. Paul, and R. K. Hanson, Quantitative Imaging of Temperature Fields in Air Using Planar Laser-Induced Fluorescence of $\mathrm{O}_{2}$, Opt. Lett. 12, 75 (1987).
[41] J. M. Seitzman, G. Kychakoff, and R. K. Hanson, Instantaneous Temperature Field Measurements Using Planar Laser-Induced Fluorescence, Opt. Lett. 10, 439 (1985).

[42] J. C. McDaniel, B. Hiller, and R. K. Hanson, Simultaneous Multiple-Point Velocity Measurements Using LaserInduced Iodine Fluorescence, Opt. Lett. 8, 51 (1983).

[43] P. H. Paul, M. P. Lee, and R. K. Hanson, Molecular Velocity Imaging of Supersonic Flows Using Pulsed Planar LaserInduced Fluorescence of NO, Opt. Lett. 14, 417 (1989).

[44] J.-U. Kim, Y. J. Kim, and B. Hong, Planar Laser-Induced Fluorescence (PLIF) Measurements of a Pulsed Electrothermal Plasma Jet, KSME Int. J. 15, 1808 (2001).

[45] A. D. Sappey and T. K. Gamble, Laser-Fluorescence Diagnostics for Condensation in Laser-Ablated Copper Plasmas, Appl. Phys. B 53, 353 (1991).

[46] Yu. Kudryavtsev et al., A New In-Gas-Laser Ionization and Spectroscopy Laboratory for Off-Line Studies at $K U$ Leuven, Nucl. Instrum. Methods Phys. Res., Sect. B 376, 345 (2016).

[47] E. Mogilevskiy, Horn Cell Losses Structure, Technical Report KU Leuven, Instituut voor Kern- en Stralingsfysica, 2013.

[48] S. De Jesus Teixeira, Characterization of a High-Power High-Repetition-Rate Laser System for Atomic and Nuclear Structure Studies, Master's thesis, Université de Liegè, 2015.

[49] COMSOL MulTiPHYSICS, https://www.comsol.com/.

[50] G. Sanna and G. Tomassetti, Introduction to Molecular Beams Gas Dynamics (Imperial College Press, London, 2005).

[51] R. L. Kurucz and B. Bell, Atomic Line Data Kurucz CDROM No. 23.(Smithsonian Astrophysical Observatory), https://www.cfa.harvard.edu/amp/ampdata/kurucz23/sekur .html (1995).

[52] J. J. Olivero and R. L. Longbothum, Empirical Fits to the Voigt Line Width: A Brief Review, J. Quantum Spectrosc. Radiat. Transf. 17, 233 (1977).

[53] T. Sonoda, T. E. Cocolios, J. Gentens, M. Huyse, O. Ivanov, Yuri Kudryavtsev, D. Pauwels, P. Van den Bergh, and P. Van Duppen, The Laser Ion Source Trap (LIST) Coupled to a Gas Cell Catcher, Nucl. Instrum. Methods Phys. Res., Sect. B 267, 2918 (2009).

[54] N. Allard and J. Kielkopf, The Effect of Neutral Nonresonant Collisions on Atomic Spectral Lines, Rev. Mod. Phys. 54, 1103 (1982).

[55] A. Bielski, R. Bobkowski, and J. Szudy, Power-Law Dependence of the Pressure Broadening of Spectral Lines on Temperature, Astron. Astrophys. 208, 357 (1989).

[56] W. Gins, SATLAS: Statistical Analysis Toolbox for Laser Spectroscopy, https://zenodo.org/record/33291.

[57] G. Hermann, G. Lasnitschka, C Schwabe, and D. Spengler, Hyperfine Structures and Isotopic Shifts of the Cu DI and D2 Lines Measured by High-Resolution Laser Fluorescence Spectroscopy with a Collimated Atomic Beam, Spectrochim. Acta B Atom. Spectros. 48, 1259 (1993).

[58] P. L. Owen and C. K. Thornhill, The Flow in an AxiallySymmetric Supersonic Jet from a Nearly-Sonic Orfice into a Vacuum, Aeronautical Research Council Reports and Memoranda, 1948, http://naca.central.cranfield.ac.uk/ reports/arc/rm/2616.pdf. 
[59] H. Ashkenas and F. S. Sherman, Rarefied Gas Dynamics (Academic Press, New York, 1966), p. 94.

[60] C. A. Stearns, F. J. Kohl, G. C. Fryburg, and R. A. Miller, A High Pressure Modulated Molecular Beam Mass Spectrometric Sampling System, NASA Technical Memorandum No. 73720, 1977, https://ntrs.nasa.gov/search.jsp? $\mathrm{R}=19770025298$.

[61] T. Matsuda, U. Yoshikuni, I. Ryuji, and S. Keisuke, Numerical and Experimental Studies on Choked Underexpanded Jets, in Proceedings of the 19th AIAA Fluid Dynamics, Plasma Dynamics, and Lasers Conference, 1987, https://doi .org/10.2514/6.1987-1378.

[62] J. A. Wilkes, C. E. Glass, P. M. Danehy, and R. J. Nowak, Fluorescence Imaging of Under Expanded Jets and Comparison with CFD, in Proceedings of the 44th AIAA Aerospace Sciences Meeting and Exhibit, 2006, Vol. 910, DOI: 10.2514/6.2006-910.

[63] M. M. Orescanin and J. M. Austin, Exhaust of Underexpanded Jets from Finite Reservoirs, J. Propulsion Power 26, 744 (2010).

[64] H. Murphy, The Effects of Source Geometry on Free Jet Expansions, Ph. D. thesis, University of California, San Diego, 1984.

[65] D. R. Miller, Atomic and Molecular Beam Methods (Oxford University Press, New York, 1988).
[66] A. G. Hansen, Fluid Mechanics, Series in Thermal and Transport Sciences (Wiley, New York, 1967).

[67] J. B. Anderson, R. P. Andres, and J. B. Fenn, Advances in Chemical Physics: Molecular Beams (John Wiley and Sons, Inc., Hoboken, NJ, 1966), Vol. 10, p. 255.

[68] K. Bier and O. Hagena, Influence of Shock Waves on the Generation of High-Intensity Molecular Beams by Nozzles, Technical Report Kernforschungszentrum G.m.b.H., Karlsruhe, 1963, https://ntrs.nasa.gov/search .jsp?R=19630011703.

[69] S. Raeder et al., Developments Towards In-Gas-Jet Laser Spectroscopy Studies of Actinium Isotopes at LISOL, Nucl. Instrum. Methods Phys. Res., Sect. B 376, 382 (2016).

[70] G. Grossir, Longshot Hypersonic Wind Tunnel Flow Characterization and Boundary Layer Stability Investigations, Ph.D. thesis, Université Libre de Bruxelles, 2015.

[71] Signal Processing with OriginLab: Savitzky-Golay Method, https://www.originlab.com/doc/Origin-Help/Smoothing.

[72] S. Raeder et al., Probing Sizes and Shapes of Nobelium Isotopes by Laser Spectroscopy, Phys. Rev. Lett. 120, 232503 (2018). 